\title{
MASTER
}

\section{The NJOY Nuclear Data Processing System, Volume IV: The ERRORR and COVR Modules}

\author{
IA- -9303-M-Vol .4
}

\author{
D. W. Muir
}

DE 86007203

R. E. MacFarlane

\section{DISCLAIMER}

This report was prepared as an eccomat of work sponeored by an agency of the United States Government. Neither the Unitod States Governmeat nor any ayeacy thereof, nor any of their employees, makes any warranty, express or imaplied, or aneumes any lezal liability or reeponaibility for the accuracy, completeaces, or uefulness of any information, apparatus, product, or procese dieclosed, or represents that its we would not infrimpe privately owned rights. Reforence herein to any specific commercial product, process, of service by trade name, trademark, manufucturer, or otherwive does nol mecemarily conatitute or inply its endorsemeat, recommendation, or favoring by the United States Government or any arency thereof. The views and opinions of authors expresed herein do not necesearily state or reflect those of the United States Government or any secucy theroof. 
THE NJOY NUCLEAR DATA PROCESSING SYSTEM, VOLUME IV:

THE ERRORR AND COVR MODULES

by

D. W. Muir and R. E. MacFarlane

\begin{abstract}
The NJOY nuclear data processing system is a comprehensive computer code package for producing cross sections and related nuclear parameters from ENDF/B evaluated nuclear data. This volume provides detailed descriptions of the NJOY modules ERRORR and COVR, which are concerned with the covariances (uncertainties and correlations) of multigroup cross sections and fission $\bar{v}$ values.
\end{abstract}

XVIII. INTRODUCTION TO VOLUME IV

The NJOY nuclear data processing system is a comprehensive computer code package for producing pointwise and multigroup cross sections, and related quantities such as multigroup covariances, starting from ENDF/B-IV and - $V$ evaluated nuclear data. A concise description of the code system and references to the ancestors of NJOY are given in Vol. I of this report. Volume II provides more detailed discussions of the theory and methods used in four of the modules that prepare pointwise cross-section data. Volume III provides similar documentation of the theory and methods used in the two modules that prepare multigroup cross sections for neutron and photon transport and effects. This volune describes the two NJOY modules concerned with covariances of multigroup cross sections and fission $\bar{v}$ values. Revisions and extensions up to October 1985 are included in the modules described in this report.

The following chapter, Chapter XIX, describes the ERRORR module, which produces multigroup covariances from evaluated data in ENDF/B format. Chapter XX then describes the COVR module, which post-processes the output of ERRORR, either to produce covariance libraries for sensitivity systens or to produce publication quality plots of covariance data. 
XIX. ERRORR

\section{A. Introduction}

After an evaluator has completed his review of the available measurements of various nuclear data $\left\{\sigma_{1}, \sigma_{2}, \sigma_{3}, \cdots\right\}$, and after he has completed his theoretical analysis, he will have formed an opinion (at least a subjective one) of the joint probability distribution of the data examined; that is, the probability

$$
P\left(\sigma_{1}, \sigma_{2}, \cdots\right) d \sigma_{1} d \sigma_{2} \cdots
$$

that the true value of $\sigma_{1}$ lies in the range $\left(\sigma_{1}, \sigma_{1}+d \sigma_{1}\right)$, and that $\sigma_{2}$ lies in the range $\left(\sigma_{2}, \sigma_{2}+d \sigma_{2}\right)$, etc.

In the early versions of $\mathrm{ENDF} / \mathrm{B}$, only the first moments of this probability distribution (mean values) could be included in the numerical data files. However, beginning with ENDF/B-IV and expanding significantly in ENDF/B-V, the second moments of the data probability distributions have been included in many of the files. As discussed in Section $B$, these second moments (data covariances) contain information on the uncertainty in individual data, as well as the correlations that may exist.

These data covariances have many applications. For example, they can be combined with sensitivity coefficients to obtain the uncertainty (due to the data) in calculated quantities of applied interest. ${ }^{1}$ This information can be used, in turn, to judge the adequacy of the data for the specified application.

The availability of data covariances also makes it possible to use the generalized method of least squares to improve the data in question, after new integral or differential measurements have been performed. ${ }^{2}$ The least squares method requires only data covariances (not the full probability distribution), and the improved, or adjusted, data are guaranteed to have the smallest possible uncertainties, regardless of the actual shape of the underlying probability distribution function, $\mathrm{P}\left(\sigma_{1}, \sigma_{2}, \cdots\right)$. (See Ref. 3.) Thus, the ENDF/B covariance files contain, in about as compact a form as possible, a statement about the quality of the data, as well as the information needed to carry out future improvements on an objective basis.

In many of these applications, it is necessary to begin by converting energy-dependent covariance information in ENDF/B format ${ }^{4}$ into multigroup form. This task can be performed conveniently in the $\mathrm{NJOY}^{5,6}$ environment using the 
ERRORR module. In particular, ERRORR calculates the uncertainty in infinitely dilute multigroup cross sections (or multigroup $\bar{v}$ values), as well as the associated correlation coefficients. These data are obtained by combining absolute or relative covariances from ENDF/B Files 31, 32, and 33 with crosssection or $\bar{v}$ data from an existing processed cross-section library, either containing resonance-reconstructed "point" data or containing multigroup data from the GROUPR module of NJOY. ERRORR is coded to treat all approved ENDF/B-IV and $-V$ covariance formats, up to and including two recent format modifications, namely, "lumped-partial" covariances and a new covariance "law," LB=6.

The nethodology of ERRORR assumes that the weighting flux used to convert energy-dependent cross sections into multigroup averages is free of uncertainty. In calculating the effect of uncertainty in resonance parameters on the multigroup cross sections, it is further assumed that the entire resonance area is contained in the energy group that contains the peak. Finally, in cases in which the cross-section information is obtained from a multigroup library, it may be necessary to make assumptions about the shape of the cross section and the veight function within certain input energy groups, as discussed below in Section E. These are the only approximations or assumptions inherent to the program.

\section{B. Definitions of Covariance-Related Quantities}

For convenient reference in discussing the methodology and input requisements of the ERRORR module, we next review the basic definitions of covariancerelated quantities. Let $x$ represent an item of data in an ENDF/B evaluation, such as the cross section for a particular reaction at a particular energy $E_{X}$ or, perhaps, the capture width of a particular resonance; and let $y$ be another item of data. Let $x_{0}$ and $y_{0}$ be the evaluated values of $x$ and $y$, respectively;

$$
\mathbf{x}_{0} \equiv E[\mathrm{x}]
$$

and

$$
\mathrm{y}_{0} \equiv \mathrm{E}[\mathrm{y}]
$$

Here $E$ is the expectation operator, which performs an average over the joint probability distribution of $x$ and $y$. The second moment of this distribution is called the covariance of $x$ with $y$;

$$
\operatorname{cov}(x, y) \equiv E\left[\left(x-x_{0}\right)\left(y-y_{0}\right)\right]
$$


The covariance is a measure of the degree to which $x$ and $y$ are both affected by the same sources of error. The covariance of $x$ with itself is called the variance of $x$;

$$
\operatorname{var}(x) \equiv \operatorname{cov}(x, x)=E\left[\left(x-x_{0}\right)^{2}\right]
$$

The more familiar standard deviation $\Delta x$ (also called the "uncertainty") is simply

$$
\Delta x \equiv[\operatorname{var}(x)]^{\frac{3}{2}}=[\operatorname{cov}(x, x)]^{\frac{3}{2}} .
$$

The correlation between $x$ and $y$ (also called the correlation coefficient) is defined as

$$
\operatorname{corr}(x, y) \equiv \frac{\operatorname{cov}(x, y)}{\Delta x \Delta y}
$$

The absolute value of the correlation coefficient is always less than or equal to unity. Another useful quantity is the relative covariance of $x$ with $y$,

$$
\operatorname{rcov}(x, y) \equiv \frac{\operatorname{cov}(x, y)}{x_{0} y_{0}}
$$

Unlike the absolute covariance $\operatorname{cov}(x, y)$, the relative covariance $r \operatorname{cov}(x, y)$ is a dimensionless quantity. Closely related to the relative covariance is the relative standard deviation,

$$
\frac{\Delta x}{x_{0}}=\frac{[\operatorname{cov}(x, x)]^{\frac{3}{2}}}{x_{0}},
$$

which, fron Eq. (7), can be written as

$$
\frac{\Delta x}{x_{0}}=[\operatorname{rcov}(x, x)]^{\frac{3}{2}}
$$

Combining Eqs. (6) and (7), we have another useful result,

$$
\operatorname{corr}(x, y)=\frac{r \operatorname{cov}(x, y)}{\left(\Delta x / x_{0}\right)\left(\Delta y / y_{0}\right)}
$$


While it is customary to speak of uncertainties and correlation coefficients as separate entities, it should be noted that these are just two different aspects of the covariance. That is, if one has a set of absolute covariances for various reactions, including the self covariance, then Eqs. (5) and (6) can be used to calculate $\Delta x$ and $\operatorname{corr}(x, y)$. Similarly, if one has a set of relative covariances, one can use Eqs. (9) and (10) to calculate $\Delta x / x_{0}$ and $\operatorname{corr}(\mathrm{x}, \mathrm{y})$.

Consider now a set of nuclear parameters $\sigma_{i}$, with uncertainties characterized by the covariances $\operatorname{cov}\left(\sigma_{i}, \sigma_{j}\right)$. Let $A$ and $B$ be two linear functions of the $\sigma_{i}$, that is

$$
\begin{aligned}
& A=\sum_{i} a_{i} \sigma_{i} \\
& B=\sum_{j} b_{j} \sigma_{j},
\end{aligned}
$$

where the $a_{i}$ and $b_{j}$ are sets of known constants. The above definitions can be used to calculate the covariances of the functions induced by those of the parameters. From Eq. (3),

$$
\begin{aligned}
\operatorname{cov}(A, B) & \left.\left.=E\left\{\underset{i}{E} a_{i} \sigma_{i}-\sum_{i} a_{i} E\left(\sigma_{i}\right)\right) \underset{j}{\left(\sum b_{j}\right.} \sigma_{j}-\sum_{j} b_{j} E\left(\sigma_{j}\right)\right)\right\} \\
& =\sum_{i, j} a_{i} b_{j} E\left\{\left(\sigma_{i}-E\left(\sigma_{i}\right)\right)\left(\sigma_{j}-E\left(\sigma_{j}\right)\right)\right\},
\end{aligned}
$$

so that

$$
\operatorname{cov}(A, B)=\sum_{i, j} a_{i} b_{j} \operatorname{cov}\left(\sigma_{i}, \sigma_{j}\right)
$$

This result, called the "propagation-of-errors" formula, is fundamental to the subject of multigroup processing of ENDF/B covariance data and will be referenced frequently in later sections of this report.

C. Structure of ENDF/B Files 31 and 33: Energy Dependent Data

Data in $\mathrm{ENDF} / \mathrm{B}$ are stored in various numbered "files," where the file number depends on the type of information contained. For example, the covariances of $\bar{v}(E)$ (the average number of neutrons per fission, which is a function of the incident neutron energy) are stored in File 31 , where the possible "reaction" 
types are prompt $\bar{v}$, delayed $\bar{v}$, and total $\bar{v}$. File 32 describes the uncertainty in the shape and area of individual resonances. File 33 contains the covariances of energy-dependent cross sections. Within the resolved-resonance energy range, File 33 includes any "long-range" covariances in the reconstructed cross sections, such as covariance components that are highly correlated over several neighboring resonances. The structure of Files 31 and 33 are identical and will be described first.

Files 31 and 33 describe the covariances of energy dependent data. The full energy dependence of a cross section $\sigma(E)$, for example, is described in the ENDF/B File 3 by specifying the cross-section values at a relatively small number of energy points and then providing a set of interpolation "laws" to be used in reconstructing the actual cross section at any intermediate energy. Somewhat the same philosophy is used to describe the two-dimensional energy dependence of data covariances. That is, one specifies a set of numerical data and a set of formulae, which together can be used to compute $\operatorname{cov}(x, y)$ for any desired pair of energies, $E_{x}$ and $E_{y}$. Although the interpolation laws are presently restricted to the simple forms described below, it is not true (as it is sometimes stated) that ENDF/B contains multigroup covariances. The expression "multigroup covariance" usually refers to the covariance of one multigroupaveraged quantity with another averaged quantity, whereas ENDF contains the covariances between energy point data.

Files 31 and 33 of an evaluation for material MAT are divided into "sections," indexed by the reaction type MT. A section (MAT,MT) is further subdivided into "subsections." As described in Ref. 4, a subsection is the repository for all explicit statements of the two-dimensional energy dependence of the covariances of reaction (MAT,MT) with another reaction (MAT1,MT1), Because the covariance is symetric, a subsection with MAT1 = MAT and MT1 < MT would be redundant with a subsection in an earlier section, and such data are, by convention, omitted from the ENDF files.

Subsections are further divided into "sub-subsections." Two different types of sub-subsections are used in ENDF/B-V. (Although ERRORR also treats data covariances in the earlier ENDF/B-IV format, this is of little practical interest since only three covariance evaluations were released in ENDF/B-IV format.) "NI-type" sub-subsections are used to express covariances explicitly, while "NC-type" sub-subsections are used to indicate the existence of connections between various data, which result in "implicit" covariance contributions for various reaction pairs. We shall return to this point later. 
Multiple NI-type sub-subsections are used to describe multiple, statistically independent sources of uncertainty for a given reaction pair. Thus, if $\operatorname{cov}(x, y)_{n}$ is the covariance computed from the data in one sub-subsection, then, because the uncertainties in different sub-subsections are uncorrelated,

$$
\operatorname{cov}(x, y)=\sum_{n=1}^{N I} \operatorname{cov}(x, y)_{n},
$$

where NI is the number of NI-type sub-subsections in the current subsection.

The numerical content of one NI-type sub-subsection consists of either one or two energy grids, a set of constants, and a parameter LB. The parameter IB governs how the energies and constants are to be used in constructing the covariance in various rectangular regions of $E_{x}-E_{y}$ space. For $L B=0,1$, and 2 , a single table containing pairs $\left(E_{i}, F_{i}\right)$ is given. For $L B=3$ and 4 , a second table $\left(E_{i}^{\prime}, F_{i}^{\prime}\right)$ is also given. For $L B=5$, a single set of energies $E_{i}$ is given, along with an associated square matrix of constants $G_{i j}$. Finally, for $L B=6$, two energy grids are given, along with an associated matrix of constants $G_{i j}^{\prime}$, which is either square or rectangular, depending on the choice of energy grids.

The table $\left(E_{i}, F_{i}\right)$ defines a function $f(E)$ that is constant in magnitude, except for discrete steps at the energies $E_{i}$,

$$
f(E) \equiv F_{i} \text {, if } E_{i} \leqq E<E_{i+1}
$$

Similarly, if there is a second table,

$$
f^{\prime}(E) \equiv F_{i}^{\prime} \text {, if } E_{i}^{\prime} \leqq E<E_{i+1}^{\prime}
$$

The $L B=5$ matrix data $G_{i j}$ also define a function,

$$
\begin{array}{r}
g\left(E_{x}, E_{y}\right) \equiv G_{i j}, \text { if } E_{i} \leqq E_{x}<E_{i+1} \\
\text { and } E_{j} \leqq E_{y}<E_{j+1},
\end{array}
$$

and similarly for $L B=6$,

$$
g^{\prime}\left(E_{x}, E_{y}\right) \equiv G_{i j}^{q}, \text { if } E_{i} \leqq E_{x}<E_{i+1}
$$




$$
\text { and } E_{j}^{\prime} \leqq E_{y}<E_{j+1}^{\prime}
$$

These functions are simply histograms in either one or two dimensions. Using the functions $f, f^{\prime}, g$, and $g^{\prime}$ thus defined, we can list the formulae, Eq. (13a$13 \mathrm{~g}$ ), which are used in $E N D F / B-V$ to specify ene:gy dependent covariances for the different allowed values of $\mathrm{LB}$. Thus, if $\mathrm{x}$ is the value of the cross section or $\bar{v}$ value for the reaction (MAT,MT) that determines the ENDF/B section, and if $y$ is that for the reaction (MAT1,MT1) that determines the subsection, then for

$$
\begin{aligned}
& \text { LB }=0, \operatorname{cov}(x, y)_{n}=f\left(E_{x}\right) \delta\left(E_{x}, E_{y}\right) \\
& L B=1, \operatorname{rcov}(x, y)_{n}=f\left(E_{x}\right) \delta\left(E_{x}, E_{y}\right) \\
& L B=2, \operatorname{rcov}(x, y)_{n}=f\left(E_{x}\right) f\left(E_{y}\right) \\
& L B=3, \operatorname{rcov}(x, y)_{n}=f\left(E_{x}\right) f^{\prime}\left(E_{y}\right) \\
& L B=4, \operatorname{rcov}(x, y)_{n}=f\left(E_{x}\right) \delta\left(E_{x}, E_{y}\right) f^{\prime}\left(E_{x}\right) f^{\prime}\left(E_{y}\right) \\
& L B=5, \operatorname{cov}(x, y)_{n}=8\left(E_{x}, E_{y}\right) \\
& L B=6, \operatorname{rov}(x, y)_{n}=8^{\prime}\left(E_{x}, E_{y}\right) .
\end{aligned}
$$

The symbol $\delta\left(E_{x}, E_{y}\right)$ has the following meaning: $\delta\left(E_{x}, E_{y}\right)=1$ if $E_{x}$ and $E_{y}$ fall in the same energy interval of the first table $\left(E_{i}, F_{i}\right)$, and $\delta\left(E_{x}, E_{y}\right)=0$ otherwise.

NC-type sub-subsections, which describe covariances indirectly, are used in several evaluation situations, which are "flagged" by different values of the parameter LTY. The first situation, with LTY $=0$, occurs when the covariances of a given reaction $M T$, both with itself and with other reactions, can be inferred in an energy range $(E 1, E 2)$ through the use of a cross-section "derivation relation,"

$$
x(\mathrm{MAT}, \mathrm{MT} ; \mathrm{E})=\sum_{i} C_{i} \times(\mathrm{MAT}, \mathrm{MT} ; \mathrm{E})
$$

This approach can be used only if the covariances of all of the reactions on the right-hand side of Eq. (14) are given directly (that is, using only NI-type sub-subsections) throughout the specified energy range. The energy boundaries $E 1$ and $E 2$, the constants $C_{i}$, and the reaction identifiers HT $_{i}$ are specified in an NC-type sub-subsection with LTY $=0$. This format is widely used in ENDF/B, and it makes possible the elimination of large volunes of otherwise redundant 
data. Unfortunately, it also introduces considerable complexity in the multigroup processing, as discussed in Section G. For example, the presence of this one short sub-subsection affects the calculation of the covariances for many different reaction pairs, such as $x$ (MAT,MT) with $x\left(\right.$ MAT, MT $\left._{i}\right)$.

Less widely used are NC-type sub-subsections with LTY $=1$. These are employed when a reaction MT in material MAT is evaluated in some energy range $(E 1, E 2)$ as a ratio to a standard reaction MTS in some other material MATS in ENDF/B. That is,

$$
x(\operatorname{MAT}, \mathrm{MT} ; E)=R(E) \times(\text { MATS }, \text { MTS } ; E) .
$$

The uncertainty of $R$ is almost never correlated with that of $x$ (MATS,MTS). Because of this, the relative uncertainty in $R$ is just one independent component of the relative uncertainty in x(MAT,MT), and it is described using normal NI-type sub-subsections. On the other hand, the contribution from uncertainty in $x$ (MATS, MTS) is represented with an NC-type sub-subsection with LTY $=1$, which contains only E1, E2, MATS, and MTS. The actual numerical covariance information must be read from the evaluation for the standard material MATS, which usually resides on an entirely different ENDF/B tape. An NC-type subsubsection with LTY $=1$, which occurs in a given subsection (MAT,MT;MAT,MT), affects the calculation of the covariances only for the current reaction pair (reaction MT with itself) and in this respect is more like an Ni-type sub-subsection than an NC-type sub-subsection with LTY $=0$. This similarity is exploited in the processing of ratio covariances, as discussed in Section $H$. An NC-type sub-subsection with LTY $=2$ is used, in a similar way, to describe the covariances of $x$ (MAT,MT) with $x$ (MATS,MTS). As in the LTY $=1$ case, an LTY $=2$ sub-subsection contains only E1, E2, MATS, and MTS.

An NC-type sub-subsection with LTY $=3$ is included in material MATS to describe the (redundant) covariances of $x$ (MATS,MTS) with $x$ (MAT,MT). The numerical information contained here is the same as for LTY $=1$ and 2. As discussed in Section $\mathrm{H}$, an important function of LTY $=3$ data is to help locate reactions other than (MAT,MT) that have been measured relative to the same standard (MATS, MTS) . 


\section{Resonance-Parameter Formats; File 32}

In File 32, the covariances of individual resonance parameters are given. At present, use of File 32 is restricted to resonance-parameter evaluations eraploying the single-level and multi-level Breit-Wigner formalisms only. For either formalism, the parameters treated in File 32 are the resonance energy $E_{r}$, the neutron width $\Gamma_{n}$, the radiative capture width $\Gamma_{\gamma}$, the fission width $\Gamma_{f}$, and the total angular momentum $J$. All cross-parameter relative covariances, such as $\operatorname{rcov}\left(\Gamma_{n}, \Gamma_{\gamma}\right)$, are included, with the exception of the covariances of $E_{r}$ with the remaining parameters, which are assumed to be negligible. Cross-resonance covariances, such as $\operatorname{cov}\left(E_{r}^{i}, E_{r}^{j}\right)$, where $i$ and $j$ refer to different resonances, are not included.

If cross-resonance parameter covariances are significant, the only possibility open to the evaluator at present is to convert this information into the form of energy-dependent covariances of the reconstructed cross sections, $\sigma(E)$, and to include this information in File 33 .

E. Calculation of Multigroup Fluxes, Cross Sections, and Covariances on the

Union Grid

As mentioned before, the main function of the ERRORR module is to calculate the uncertainty in group-averaged cross sections at infinite dilution due to uncertainty in the ENDF/B "point" data. In this and the following sections, we describe the procedures used in performing this task.

In order to proceed, it is necessary to introduce into the discussion three different energy grids, namely, the user's grid, the ENDF/B grid, and the union grid. The relationship of these three grids is shown in Fig. 1. The user's grid is the multigroup structure in which the output multigroup covariances are to be produced. The ENDF/B grid is the collection of energies obtained (in SUBROUTINE GRID) by forming the union of (a) all energy "lists" appearing in any NI-type sub-subsection of any subsection to be processed in the current ENDF/B material, and (b) all energy pairs used to define the range

$$
\begin{aligned}
& \text { User's Grid | } \quad \Phi_{1}, \mathrm{x}_{1} \quad\left|\quad \Phi_{2}, \mathrm{x}_{2} \quad\right| \\
& \text { ENDF/B Grid } \mid \begin{array}{lllll}
F_{1} \mid & F_{2} & \mid & F_{3} \mid
\end{array} \\
& \text { Union Grid }\left|\phi_{1}, x_{1}\right| \phi_{2}, x_{2}\left|\phi_{3}, x_{3}\right| \phi_{4}, x_{4} \mid
\end{aligned}
$$

Fig. 1. Illustration of energy grid relations. 
of efiectiveness of any NC-type sub-subsection of any subsection to be processed. The union grid is simply the union of the user's and FNDF/B grids. The utility of the union grid is that (a) the covariances are particularly simple to calculate in this grid, as discussed below, and (b) the multigroup covariances needed by the user are then easily obtained by a straightforward collapse from this grid to the user's grid. In fact, the design of the current ENDF/B covariance format was strongly influenced by the desire to employ this particular procedure for multigroup processing.

After the union grid is formed in SUBROUTINE UNIONG, the cross sections $x(E)$ and weighting $f l u x \phi(E)$ are integrated to produce $x_{I}$ and $\phi_{I}$, multigrouped on this grid. If point cross sections are supplied, an exact integration is done in SUBROUTINE GRPAV. If, on the other hand, a multigroup cross-section "library" is supplied, then SUBROUTINE COLAPS is used. If a library group is subdivided by a union-group boundary, then over the span of that library group, the unknown energy dependences of $x(E)$ and $\phi(E)$, which are needed to calculate $x_{I}$ and $\phi_{I}$, are approximated in SUBROUTINE COLAPS by corstants. Normally, the effect of this approximation is not large and, in any case, can be reduced by increasing the number of groups in the input library.

We next consider the theoretical basis for the calculation of union-grid multigroup covariances, as performed in SUBROUTINE COVCAL. By definition, $x_{I}$ is just the average of $x(E)$ over union group $I$,

$$
x_{I} \equiv \frac{\int_{\operatorname{group} I}(E) \times(E) d E}{\int_{\operatorname{group} I} \phi(E) d E}
$$

where $\phi(E)$ is the flux "model" assumed for the multigroup calculations. Let $y_{J}$ denote the average of $y(E)$ over union group $J$.

Let us imagine that these groups are subdivided into many subintervals of infinitesimal width, so that in the $i$-th subinterval of group $I$, for example, $x(E)$ can be well approximated by the constant $x_{i} \cdot$ By this device, the integrals that define $x_{I}$ and $y_{J}$ can be converted to discrete sums;

$$
x_{I}=\frac{\sum_{i \varepsilon I} \phi_{i} x_{i}}{\phi_{I}}=\sum_{i \varepsilon I} \alpha_{I i} x_{i}
$$

where 


$$
\begin{aligned}
& \phi_{i}=\underset{\text { subinterval } i}{\int} \phi(E) d E \\
& \phi_{I}=\sum_{i \varepsilon I} \phi_{i}=\int_{\text {group } I} \phi(E) d E,
\end{aligned}
$$

and

$$
\alpha_{I i} \equiv \frac{\phi_{i}}{\phi_{I}}
$$

From these definitions, clearly

$$
\sum_{i \varepsilon I} \alpha_{I i}=1
$$

Similarly for $y$,

$$
y_{J}=\sum_{j \in J}^{\Sigma} \alpha_{J j} y_{j}
$$

and

$$
\sum_{j \varepsilon J} \alpha_{J j}=1
$$

The methodology of ERRORR assunes that $\phi(E)$ in Eq. (16) is free of uncertainty. Under this assumption, the terms $\alpha_{I i}$ and $\alpha_{J j}$ are simply known constants. The covariance of $x_{I}$ with $y_{J}$ can then be calculated using the propagation-of-errors formula, Eq. (11), together with Eqs. (17) and (19).

$$
\begin{aligned}
\operatorname{cov}\left(x_{I}, y_{J}\right) & =\sum_{\substack{i \varepsilon I \\
j \varepsilon J}} \alpha_{I i} \alpha_{J j} \operatorname{cov}\left(x_{i}, y_{j}\right) \\
& =\sum_{\substack{i \varepsilon I \\
j \varepsilon J}} \alpha_{I i} \alpha_{J j} \sum_{n} \operatorname{cov}\left(x_{i}, y_{j}\right)_{n},
\end{aligned}
$$

where the sumation over $n$ results from the different independent contributions to the ENDF/B "point" covariances coming from the different NI-type sub-subsections. Changing the order of sumnation, we obtain

$$
\operatorname{cov}\left(x_{I}, y_{J}\right)=\sum_{n} \operatorname{cov}\left(x_{I}, y_{J}\right)_{n},
$$

where

$$
\operatorname{cov}\left(x_{I}, y_{J}\right)_{n}=\sum_{\substack{i \varepsilon I \\ j \varepsilon J}} \alpha_{I i} \alpha_{J j} \operatorname{cov}\left(x_{i}, y_{j}\right)_{n} .
$$


To evaluate the sum in Eg. (22), we make use of the fact that union groups $I$ and $J$ do not cross any ENDF/B grid boundaries. Recalling the discussion of NI-type sub-subsections in Section $C$, there are only two possibilities for the energy dependence of the covariance between ENDF/B grid points: thus, over the limits of the sum, either $\operatorname{cov}\left(x_{i}, y_{j}\right)_{n}$ is independent of $i$ and $j$ (if $L B=0$ ) or $r \operatorname{cov}\left(x_{i}, y_{j}\right)_{n}$ is independent of $i$ and $j$ (if LB $>0$ ). We consider first the constant-absolute-covariance case, $L B=0$. In this case, Eq. (22) can be rewritten as follows:

$$
\operatorname{cov}\left(x_{I}, y_{J}\right)_{n}=\operatorname{cov}(x, y)_{n} \sum_{\substack{i \varepsilon I \\ j \varepsilon J}} \alpha_{I i} \alpha_{J j}=\operatorname{cov}(x, y)_{n}\left(\sum_{i \varepsilon I} \alpha_{I i}\right)\left(\sum_{j \varepsilon J} \alpha_{J j}\right)
$$

Invoking Eqs. (18) and (20), we obtain

$$
\operatorname{cov}\left(x_{I}, y_{J}\right)_{n}=\operatorname{cov}(x, y)_{n} \quad(L B=0) .
$$

For all other LB-values, the relative covariance is constant, so we rewrite Eq. (22) in the form

$$
\begin{aligned}
\operatorname{cov}\left(x_{I}, y_{J}\right)_{n} & =\sum_{\substack{i \varepsilon I \\
j \varepsilon J}} \alpha_{I i} \alpha_{J j} x_{i} x_{j} \operatorname{rcov}\left(x_{i}, x_{j}\right)_{n} \\
& =\operatorname{rcov}(x, y)_{n} \sum_{\substack{i \varepsilon I \\
j \varepsilon J}}\left(\alpha_{I i} x_{i}\right)\left(\alpha_{J j} y_{j}\right) \\
& =\operatorname{rcov}(x, y)_{n}\left(\sum_{i \varepsilon I} \alpha_{I i} x_{i}\right)\left(\sum_{j \varepsilon J} \alpha_{J j} y_{j}\right) .
\end{aligned}
$$

Substituting here from Eqs. (17) and (19), we obtain

$$
\operatorname{cov}\left(x_{I}, y_{J}\right)_{n}=x_{I} y_{J} r \operatorname{cov}(x, y)_{n} \quad(L B>0) .
$$

The final union-group multigroup covariance is obtained by inserting these results, Eqs. (23) and (24), back into Eq. (21).

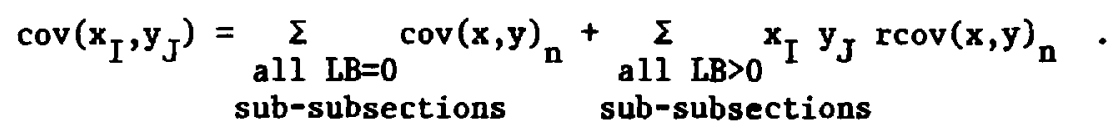


The quantities $\operatorname{cov}(x, y)_{n}$ and $r \operatorname{cov}(x, y)_{n}$ here are simply the "point" energy covariances from the ENDF/B covariance file, as described in Eqs. (12) and (13). Eq. (25), then, is the basic equation used in SUBROUTINE COVCAL to calculate the desired union-group covariances.

F. Basic Strategy for Collapse to the User's Grid

The union-group fluxes are used, in SUBROUTINE SIGC, to collapse the uniongroup cross sections to the coarser user's grid. Changing notation slightly, let us denote by $x_{I}(a)$ the cross section in union group $I$ for reaction $a$, and similarly let $X_{K}(a)$ be the cross section in user group $K$ for the same reaction. In complete analogy with Eq. (17)

$$
\mathrm{X}_{\mathrm{K}}(\mathrm{a})=\frac{\sum_{I \varepsilon \mathrm{K}} \phi_{I} \mathrm{x}_{I}(\mathrm{a})}{\Phi_{\mathrm{K}}}=\sum_{I \varepsilon \mathrm{K}} A_{\mathrm{KI}} \mathrm{x}_{I}(\mathrm{a}),
$$

where

$$
\Phi_{K}=\sum_{I \varepsilon K} \phi_{I}
$$

and

$$
A_{\mathrm{KI}}=\frac{\phi_{I}}{\phi_{K}}
$$

Again applying the propagation-of-errors formula,

$$
\operatorname{cov}\left[\mathrm{X}_{K}(\mathrm{a}), \mathrm{X}_{\mathrm{L}}(\mathrm{b})\right]=\sum_{\substack{I \varepsilon K \\ J \varepsilon L}} A_{K I} A_{L J} \operatorname{cov}\left[\mathrm{x}_{\mathrm{I}}(\mathrm{a}), \mathrm{x}_{\mathrm{J}}(\mathrm{b})\right]
$$

An alternative expression, obtained by simply rearranging coefficients, is

$$
\operatorname{cov}\left[X_{K}(a), X_{L}(b)\right]=\frac{1}{\Phi_{K} \phi_{L}} \sum_{\substack{I \varepsilon K \\ J \varepsilon L}} T_{I J}(a, b),
$$

where

$$
T_{I J}(a, b)=\phi_{I} \phi_{J} \operatorname{cov}\left[x_{I}(a), x_{J}(b)\right]
$$


Eqs. (30) and (31) provide the basic framework in the ERRORR module for the production of multigroup covariances in the coarse user's-group structure. The final step, if the user requests it, is to corvert the absolute covariances from $\mathrm{Eq}$. (30) to relative form,

$$
\operatorname{rcov}\left[X_{K}(a), X_{L}(b)\right]=\frac{\operatorname{cov}\left[X_{K}(a), X_{L}(b)\right]}{X_{K}(a) X_{L}(b)}
$$

\section{G. Group-Collapse Strategy for Data Derived by Summation}

The procedure described in the previous section must be modified if, in some energy range, either reaction a or b is a "derived" quantity in the sense of Eq. (14) or Eq. (15). In such a case, one cannot apply Eq. (25) directly to the calculation of $\operatorname{cov}\left[x_{I}(a), x_{J}(b)\right]$, because the covariances on the right-hand side of Eq. (25) will be missing from the ENDF/B file in the affected energy region.

We consider first the case in which some cross sections are evaluated ("derived") by simply summing other evaluated cross sections, as in Eq. (14). Eq. (26) can then be rewritten as

$$
X_{K}(a)=\sum_{I \varepsilon K} A_{K I}\left\{\sum_{a I 1} c_{I}(a, c) x_{I}(c)\right\}
$$

where the quantity in curly brackets is the value of the derived cross section "a" in union group $I$, as reconstructed from the directly evaluated cross sections $x_{I}(c)$. The derivation coefficients $C_{I}(a, c)$ depend on the union-group index $I$, because the evaluator is permitted to employ different derivation strategies in different energy ranges.

To expand on this last point, suppose there are only three reactions, and suppose that the cross sections $x(1)$ and $x(2)$ rigorously sum to $x(3)$ at all energies. Further suppose that within the energy range covered by union group number 1, the cross-section evaluator has used this logical connection in order to "derive" $x(3)$, that is, to evaluate $x(3)$, by simply summing his existing evaluations for $x(1)$ and $x(2)$,

$$
x_{1}(3)=x_{1}(1)+x_{1}(2)
$$


Further suppose that in the range of union group $2, x(1)$ is "derived" by employing the same logical connection, but in a different way,

$$
x_{2}(1)=x_{2}(3)-x_{2}(2),
$$

and in union group 3, all three reactions are directly evaluated.

In this example $\varepsilon_{I}(a, c)$ has the following values:

$$
\begin{array}{lll}
c_{1}(1,1)=1 & c_{1}(1,2)=0 & c_{1}(1 ; 3)=0 \\
c_{1}(2,1)=0 & c_{1}(2,2)=1 & c_{1}(2,3)=0 \\
c_{1}(3,1)=1 & c_{1}(3,2)=1 & c_{1}(3,3)=0 \\
\hdashline c_{2}(1,1)=0 & c_{2}(1,2)=-1 & c_{2}(1,3)=1 \\
c_{2}(2,1)=0 & c_{2}(2,2)=1 & c_{2}(2,3)=0 \\
c_{2}(3,1)=0 & c_{2}(3,2)=0 & c_{3}(3,3)=1 \\
c_{3}(1,1)=1 & c_{3}(1,2)=0 & c_{3}(1,3)=0 \\
c_{3}(2,1)=0 & c_{3}(2,2)=1 & c_{3}(2,3)=0 \\
c_{3}(3,1)=0 & c_{3}(3,2)=0 & c_{3}(3,3)=1
\end{array}
$$

Note that we have rormally considered the evaluated cross sections to be "derived from themselves," $\left.c_{I}(a, c)\right|_{\text {eval }}=\delta_{a c}$. Use of this device allows us to use Eq. (32) for all reactions, regardless of whether they are "derived" or "evaluated." Note that, in every case where reaction $c$ is "derived" in group $I, C_{I}(a, c)=0$. (See dashed boxes.) This is important because it means that Eq. (32) can be treated as a linear relation involving only dependent quantities (the user-group cross sections) on the left and only independent quantities with known covariances (the "evaluated" subset of the union-group cross sections) on the right. This allows us once again to use the propagation-oferrors formula to obtain the desired user-group covariances.

$$
\begin{aligned}
& \operatorname{cov}\left[X_{K},(a), X_{L}(b)\right]=
\end{aligned}
$$

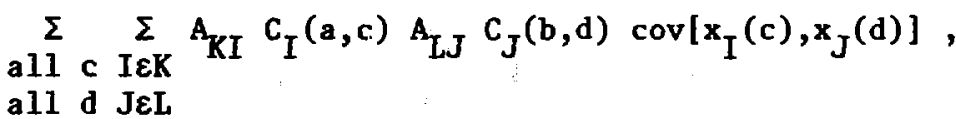

or, alternatively, 


$$
\operatorname{cov}\left[X_{K}(a), X_{L}(b)\right]=\frac{1}{\Phi_{K} \phi_{L}} \sum_{\text {all }}^{\sum} \sum_{\text {all }} \sum_{\text {IEK }}^{\sum} C_{I}(a, c) C_{J}(b, d) T_{I J}(c, d),
$$

where

$$
\mathrm{T}_{\mathrm{IJ}}(\mathrm{c}, \mathrm{d})=\phi_{\mathrm{I}} \phi_{\mathrm{J}} \operatorname{cov}\left[\mathrm{x}_{\mathrm{I}}(\mathrm{c}), \mathrm{x}_{\mathrm{J}}(\mathrm{d})\right] .
$$

In SUBROUTINE COVCAL, the flux-covariance product $T_{I J}$ is calculated for all evaluated reaction pairs and written to a "scratch" binary disk file, unit 11. (For diagnostic purposes, it is possible to change to a formatted scratch file by resetting the variable IMODE to +1 in the main program.) In the subsequent collapse to the user's group structure in SUBROUTINE COVOUT, the usergroup covariances $\operatorname{cov}\left[\mathrm{X}_{K}(\mathrm{a}), \mathrm{X}_{\mathrm{L}}(\mathrm{b})\right]$ for one specific $a-b$ reaction pair, for all "MT1" energy groups L, and for as large a range of "MT" energy groups $K$ as possible are calculated simultaneously in core.

In COVOUT, the disk file (unit 11 ) is rewound and read completely through, once in each range of "MT" groups for each a-b reaction piiir, to access the union-group covariances for the "evaluated" reactions. As tie union-group data pass through core, contributions to the user-group covariarces are accumulated using Eq. (34). To minimize the number of disk reads, the dimension of COMMON/ ESTORE/ (and the vaiue of the associated variable NAMAX) should be set as large as possible.

In the fairly common situation where cross sections $x(a)$ and $x(b)$ both are directly evaluated over the whole energy range of the data file,

$$
c_{I}(a, c)=\delta_{a c} \text {, for all I, }
$$

and

$$
c_{J}(b, d)=\delta_{b d} \text {, for all } \mathrm{J} \text {. }
$$

In this case, Eq. (34) can be greatly simplified.

$$
\begin{aligned}
& \operatorname{cov}\left[\mathrm{X}_{\mathrm{K}}(\mathrm{a}), \mathrm{X}_{\mathrm{L}}(\mathrm{b})\right]=\frac{1}{\Phi_{\mathrm{K}} \Phi_{\mathrm{L}}} \sum_{\mathrm{all}} \sum_{\mathrm{I} \varepsilon \mathrm{K}} \delta_{\mathrm{ac}} \delta_{\mathrm{bd}} \mathrm{T}_{I J}(\mathrm{c}, \mathrm{d}) \\
& \text { all d JEL } \\
& =\frac{1}{\Phi_{K} \phi_{L}} \sum_{\substack{I \varepsilon K \\
J \varepsilon L}} T_{I J}(a, b)
\end{aligned}
$$


This result, which is identical to Eq. (30), suggests a "short-cut" calculational path, which is followed in SUBROUTINE COVOUT whenever both reactions are directly evaluated. In preparation for this situation, a second copy of the COVCAL binary output file is generated when CovOUT is first called. The second copy, on unit 12 , is read only in the trivial derivation cases just described (flagged in the code by setting ISD $=1$ ). Unit 12 is not rewound before processing a given reaction pair $a-b$, since "earlier" reaction pairs on the file do not contribute to the sum in Eq. (34) in these cases. Reading of the second copy stops when the union-group covariances for reaction pair a-b are found, because the "later" reaction pairs do not contribute either.

\section{H. Processing of Data Derived from Ratio Measurements}

The treatment of implicit covariances that arise from ratio measurements, as in Eq. (15), is totally different from the treatment of data derived by summation, Eq. (14), discussed in the previous section. Before discussing the processing details, it is helpful first to review the subject of ratio evaluations generally. Returning to the " $x-y$ " notation of Section $B$, let $x\left(E_{x}\right)$ be the value of the cross section for reaction $x$ at energy $E_{x}$, and $y\left(E_{y}\right)$ the cross section for reaction $y$ at energy $E_{y}$. In some energy region $\left(L_{x}, H_{x}\right)$, suppose that the best knowledge of $x\left(E_{x}\right)$ is obtained through the application of a measured ratic, $f\left(E_{x}\right)$;

$$
x\left(E_{x}\right)=f\left(E_{x}\right) z\left(E_{x}\right) \text {, if } L_{x} \leqq E_{x} \leqq H_{x}
$$

where reaction $z$ is some well-known cross section, perhaps one of the official ENDF/B standard cross sections. Similarly, suppose $y$ is derived from the same standard, over a possibly different energy range;

$$
y\left(E_{y}\right)=g\left(E_{y}\right) z\left(E_{y}\right), \text { if } L_{y} \leqq E_{y} \leqq H_{y}
$$

By performing a first-order Taylor-series expansion and then applying the propagation-of-errors formula, one can obtain an expression for the contribution to the relative covariance $r \operatorname{cov}\left[x\left(E_{x}\right), y\left(E_{y}\right)\right]$ that is attributable to the ratio measurements. In the usual case, where the ratios $f$ and $g$ are only weakly correlated with the standard cross section $z$, the result is quite simple; 


$$
\operatorname{rcov}\left[x\left(E_{x}\right), y\left(E_{y}\right)\right]_{\text {ratio }}=\operatorname{rcov}\left[f\left(E_{x}\right), g\left(E_{y}\right)\right]+\operatorname{rcov}\left[z\left(E_{x}\right), z\left(E_{y}\right)\right]
$$

$$
\text { if } \begin{aligned}
L_{x} \leqq E_{x} \leqq H_{x} \text { and } L_{y} \leqq E_{y} \leqq H_{y} \text {, and } \\
\operatorname{rcov}\left[x\left(E_{x}\right), y\left(E_{y}\right)\right]_{\text {ratio }}=0
\end{aligned}
$$

otherwise.

Thus, in this fairly common evaluation situation, the covariance separates naturally into a part involving only the measured ratios and a part involving only the standard. Because the second contribution, $\operatorname{cov}(z, z)$, can be read directly from the NI-type sub-subsections in evaluation for the standard, it is not included explicitly in the ENDF/B subsections for the derived quantities, $\operatorname{cov}(x, x)$ or $\operatorname{cov}(x, y)$. Instead, the exiscence of this additional contribution to the covariance is signalled by the presence of an NC-type sub-subsection, as discussed in Section $C$.

The strategy adopted for processing this information in SUBROUTINE STAND is to load the NI-type sub-subsections from the evaluation of the standard into the same storage array that is used to store NI-type covariances from the evaluation for reaction $x$. From that point on, the data from the standard are handled just as if they had come from the evaluation for $x$, but with one exception. As indicated by Eq. (37), the covariance contribution $\operatorname{cov}\left[z\left(E_{x}\right), z\left(E_{y}\right)\right]$ is not added into the total covariance matrix for the current reaction pair if either $E_{x}$ or $E_{y}$ lies outside the corresponding energy "window" $\left(L_{x}, H_{x}\right)$ or $\left(L_{y}\right.$, $\mathrm{H}_{\mathbf{y}}$ ).

As discussed in Section $C$, in addition to identifying the standard reaction, the NC-type sub-subsection contains a control parameter LTY and two energies EL and EH whose significance depends on LTY. LTY is used to identify particular evaluation scenarios: reaction $x$ is the same as reaction $y$ (or, at least, they are derived from $z$ over the same energy range) (LTY $=1$ ); $y$ is identical to the standard ( $(T Y=2)$; or $x$ is identical to the standard (LTY=3). A fourth possibility, namely, that $x, y$, and $z$ are entirely distinct, cannot presently be treated with a single NC-type sub-subsection; that is, there is no LTY-value defined for this case. However, as discussed later, it is still possible to process covariances for this situation by combining information from sub-subsections in two different evaluations. For convenience, we shall refer to this fourth case as LTY $=4$. 
The interrelationship of LTY, EL, EH, and the windows $\left(\mathrm{L}_{x}, H_{x}\right.$ ) and ( $\mathrm{I}_{y}$, $H_{y}$ ) used in ERRORR for the "zeroing-out" operation, Eq. (37), is summarized below:

$$
\begin{aligned}
\operatorname{LTY}= & 1 \\
& \left(L_{x}, H_{x}\right)=\left(L_{y}, H_{y}\right)=(E L, E H) \\
\operatorname{LTY}= & 2 \\
\left(L_{x}, H_{x}\right) & =(E L, E H) \\
\left(L_{y}, H_{y}\right) & =\left(10^{-5} \mathrm{eV}, 20 \mathrm{MeV}\right) \\
\operatorname{LTY}= & 3 \\
\left(L_{x}, H_{x}\right) & =\left(10^{-5} \mathrm{eV}, 20 \mathrm{MeV}\right) \\
& \left(L_{y}, H_{y}\right)=(E L, E H) \\
\operatorname{LTY}= & 4, \\
& \left(L_{x}, H_{x}\right)=(E L, E H) \\
\left(L_{y}, H_{y}\right) & =\left(E L^{\prime}, E H^{\prime}\right) .
\end{aligned}
$$

If the user requests covariance data $\operatorname{cov}(x, y)$, where $x$ and $y$ are different and both are distinct from the standard (LTY=4), the ERRORR module obtains (EL, EH) from the ITY=2 subsection in the evaluation for $x$ that "points" to $z$. Then the covariance file for $z$ is read to obtain both (a) the explicit covariances $\operatorname{cov}(z, z)$ and $(b)$ the second energy window (EL', $E H^{\prime}$ ), the latter being found in the LTY=3 sub-subsection that "points" back to $y$.

Table I lists all ENDF/B-V reactions that contain ratio-to-standard covariance data. The symbols entered in the reaction-by-reaction matrix indicate which reactions are referenced as standards $(\stackrel{\star}{*})$, and which reaction-pairs have implicit non-zero covariauces (LTY). ERRORR will produce multigroup covariances for any of the reaction pairs in Table I that are marked with $(\not{h})$ or (LTY). The cross-material covariances (LTY=2, 3, or 4) must be requested individually using the IREAD $=2$ option (see section $K$, especially the discussion of cards 10 and 11). An attempt to process covariances for any of the cases LTY=1 through 4 without supplying a separate ENDF/B tape containing the needed standard will result in an error stop in SUBROUTINE GRID. The error diagnostic, however, will supply the details of the corrective action required for resubmitting the job. (See discussion of diagnostic messages in Section M.) 
TABLE I

COVARIANCE MATRICES AFFECTED BY RATIO MEASUREMENTS IN ENDF/B-V

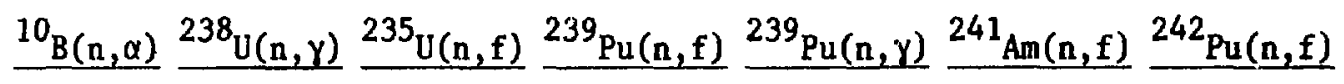

${ }^{10} \mathrm{~B}(\mathrm{n}, \alpha) \quad \div \quad 3$

${ }^{238} \mathrm{U}(\mathrm{n}, \gamma) \quad 2 \quad 1$

${ }^{235} \mathrm{U}(\mathrm{n}, \mathrm{f})$

${ }^{239} \mathrm{Pu}(\mathrm{n}, \mathrm{f})$

${ }^{239} \mathrm{Pu}(\mathrm{n}, \mathrm{\gamma})$

${ }^{241} \mathrm{Am}(\mathrm{n}, \mathrm{f})$

${ }^{242} \mathrm{Pu}(\mathrm{n}, \mathrm{f})$

3

.

$\begin{array}{ll}* & 3 \\ 2 & 1 \\ 2 & 1 \\ 2 & 4 \\ 2 & 4\end{array}$

3

3

3

1

1

4

4

4

4

4

4

1

4

41

$*$ = standard

Integer $=$ LTY value $($ see text $)$

As an example of the ratio-data capabilities of ERRORR, in Fig. 2 are shown the covariances between the fission cross sections of ${ }^{239} \mathrm{Pu}$ (reaction $\mathrm{x}$ ) and those of the important actinide ${ }^{241} \mathrm{Am}$ (reaction y). This is an LTY 4 case where $\left(\mathrm{L}_{x}, \mathrm{H}_{x}\right)=(0.2 \mathrm{MeV}, 15 \mathrm{MeV})$ and $\left(\mathrm{L}_{y}, \mathrm{H}_{y}\right)=(0.2 \mathrm{MeV}, 20 \mathrm{MeV})$. The effect of the use of two different windows is apparent ia the lower right corner of the correlation matrix. The plot itself was produced with the COVR module, which is described in Chapter $\mathrm{XX}$ of this report. 


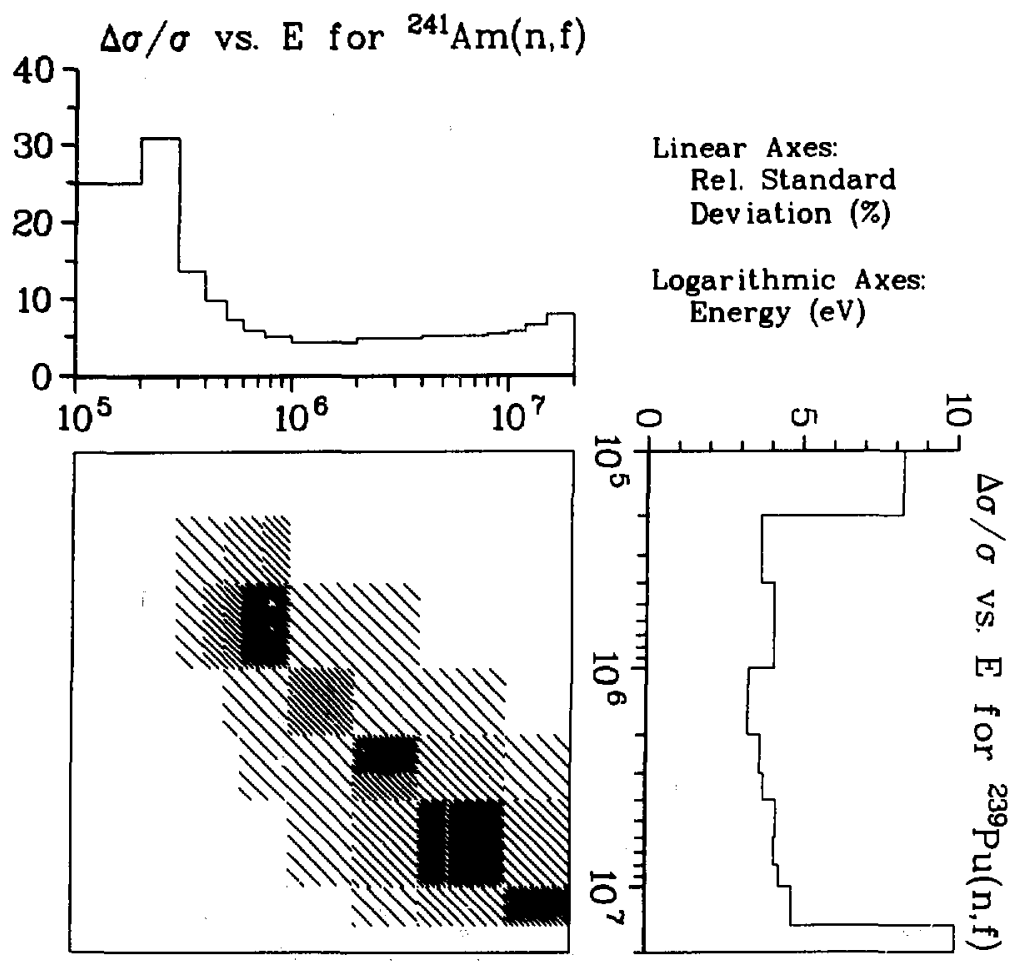

Correlation Matrix

Key:

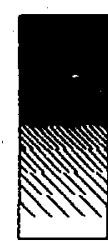

1.00
0.80
0.80
0.40
0.20
0.00

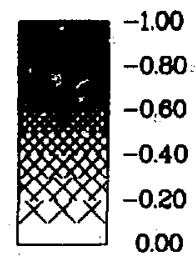

Fig. 2

Covariance data for ${ }^{239} \mathrm{Pu}(\mathrm{n}, \mathrm{f})$ with ${ }^{241} \mathrm{Am}(\mathrm{n}, \mathrm{f})$.

\section{Multigroup Processing of Resonance Parameter Uncertainties}

In some materials, and in certain energy regions, the cross-section uncertainty is dominated by the uncertainty in resolved resonance parameters. One noteworthy example is ${ }^{63} \mathrm{Cu}(\mathrm{n}, \mathrm{y}){ }^{64} \mathrm{Cu}$ (ENDF/B-V dosimetry material, MAT6435) in the energy range from $10 \mathrm{eV}$ to $15.9 \mathrm{keV}$, where the entire cross-section uncertainty is represented by means of resonance-parameter uncertainties. The same is true of ${ }^{237} \mathrm{~Np}(\mathrm{n}, \mathrm{f}$ ) (MAT6337) from 0 to $10 \mathrm{eV}$. 
In ERRORR, the resonance-parameter contribution to the uncertainty in infinite-dilution fission and capture cross sections is included automatically when cross-section covariances are processed. (See the discussion of the input option MFCOV = 33 in Section K.) Following a procedure suggested in Ref. 8, the contribution is obtained from the Breit-Wigner formulae for the fission and capture areas of a resonance, $A_{f}$ and $A_{\gamma}$. By differentiating these formulae with respect to the resonance parameters, one obtains a set of sensitivities. With these sensitivities and the covariance matrix of the parameters from MF32, one can apply the propagation-of-errors formula to obtain the covariances $\operatorname{cov}\left(A_{\gamma}, A_{\gamma}\right), \operatorname{cov}\left(A_{\gamma}, A_{f}\right)$, and $\operatorname{cov}\left(A_{f}, A_{f}\right)$. In SUBROUTINE RESCON, these results are added directly into the user-group cross-section covariances calculated from MF33.

The resonance contribution is properly weighted with the isotopic abundance and the ratio of the weight function at the resonance to the average weight in the group. It is assumed, however, that the area of a resonance lies entirely within the group that contains the resonance energy $E_{r} \cdot$ Because of this assumption, and because ENDF/B-V provides no correlations between parameters of different resonances, the calculated resonance-parameter contribution affects only the diagonal elements of the affected matrices, $\operatorname{cov}\left[\mathrm{X}_{\mathrm{K}}(\mathrm{a}), \mathrm{X}_{\mathrm{K}}(\mathrm{b})\right]$.

With the inclusion of resonance-parameter covariances, the uncertainty in the capture cross section of ${ }^{63} \mathrm{Cu}$, computed for a group that contains the large $577-\mathrm{eV}$ resonance, is $3.0 \%$, rather than zero, as it would be if the MF32 contribution were omitted.

\section{J. Processing of Lumped-Partial Covariances}

A relatively recent extension of the ERRORR mocile allows the processing of the "lumped-partial" covariance format, 9 approved by the Cross Section Evaluation Working Group in May 1981. This format allows the evaluator to specify a group of nuclear reactions and to give the uncertainty only in the sum of the cross sections for those reactions. One can, for example, replace 30 or 40 discrete-level inelastic cross sections with 5 or 6 "lumped" cross sections when constructing the covariance files. Because the volume of the covariance data varies, in general, as the square of the number of reactions, this lumping can greatly reduce the size of the files.

The first ENDF/B evaluation to employ the lumped-partial format is $P$. G. Young's evaluation ${ }^{10}$ for ${ }^{7} \mathrm{Li}$ (ENDF/B-V, Rev. 23. All covariance data for this 
evaluation have been been successfully processed into multigroup form using ERRORR. The covariances for MT854 (a single "real" level with an excitation energy of $4.63 \mathrm{MeV}$ ) with MT855 ( 6 lumped pseudo-levels, with excitation energies ranging from $4.75 \mathrm{MeV}$ to $6.75 \mathrm{MeV}$ ) have been plotted in Fig. 3. The large negative correlations along the diagonal result from the fact that, below 10 $\mathrm{MeV}$, these inelastic reactions are the major contributors to the fairly wellknown tritiun-production cross section. An upward variation in one reaction at a given energy must be accompanied by a downward change in the other. As shown

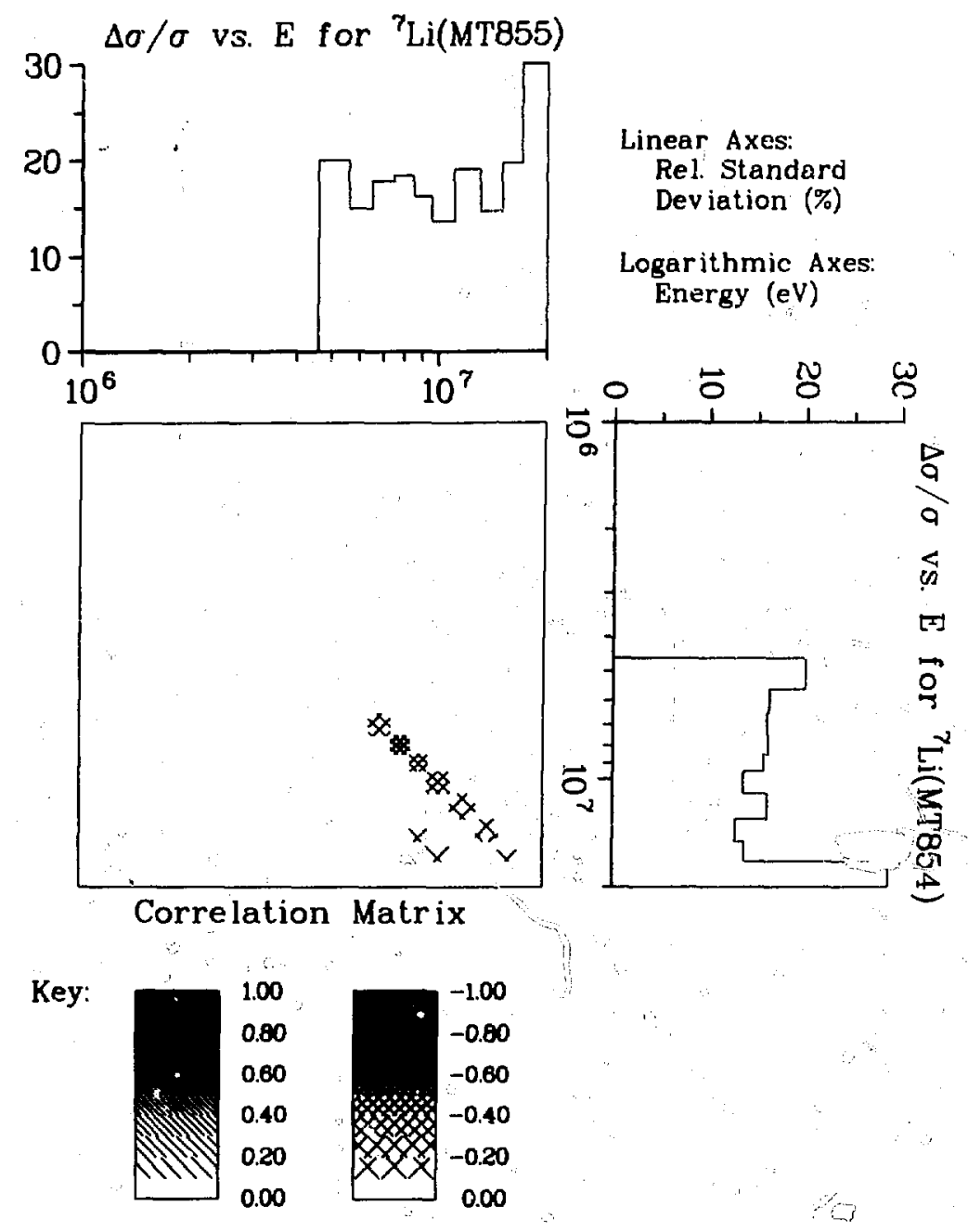

Fig. 3.

Covariance data for ${ }^{7} \mathrm{Li}\left(\mathrm{MT854)}\right.$ with ${ }^{7} \mathrm{Li}(\mathrm{MT} 855)$. 
in the plot, the magnitude of this negative correlation diminishes at higher energies, as other reactions begin to contribute significantly to the tritiumproduction cross section. Plots of this type, prepared using ERRORR and COVR, have proven to be useful tools in the validation of the covariance files of new evaluations.

\section{K. Input Instructions and Sample Input for ERRORR}

As an aid to discussions of the user input to ERRORR, we list below the input instructions that appear as comment cards at the beginning of the current. version of this module. Since the code and, hence, the instructions changc from time to time, it is always advisable to consult the comment-card instructions contained in the version of the code actually being used and not to rely on the instructions published in any document, including this one. Note that the word "tape" is used in the instructions to refer to an input or output file, regardless of the actual storage medium.

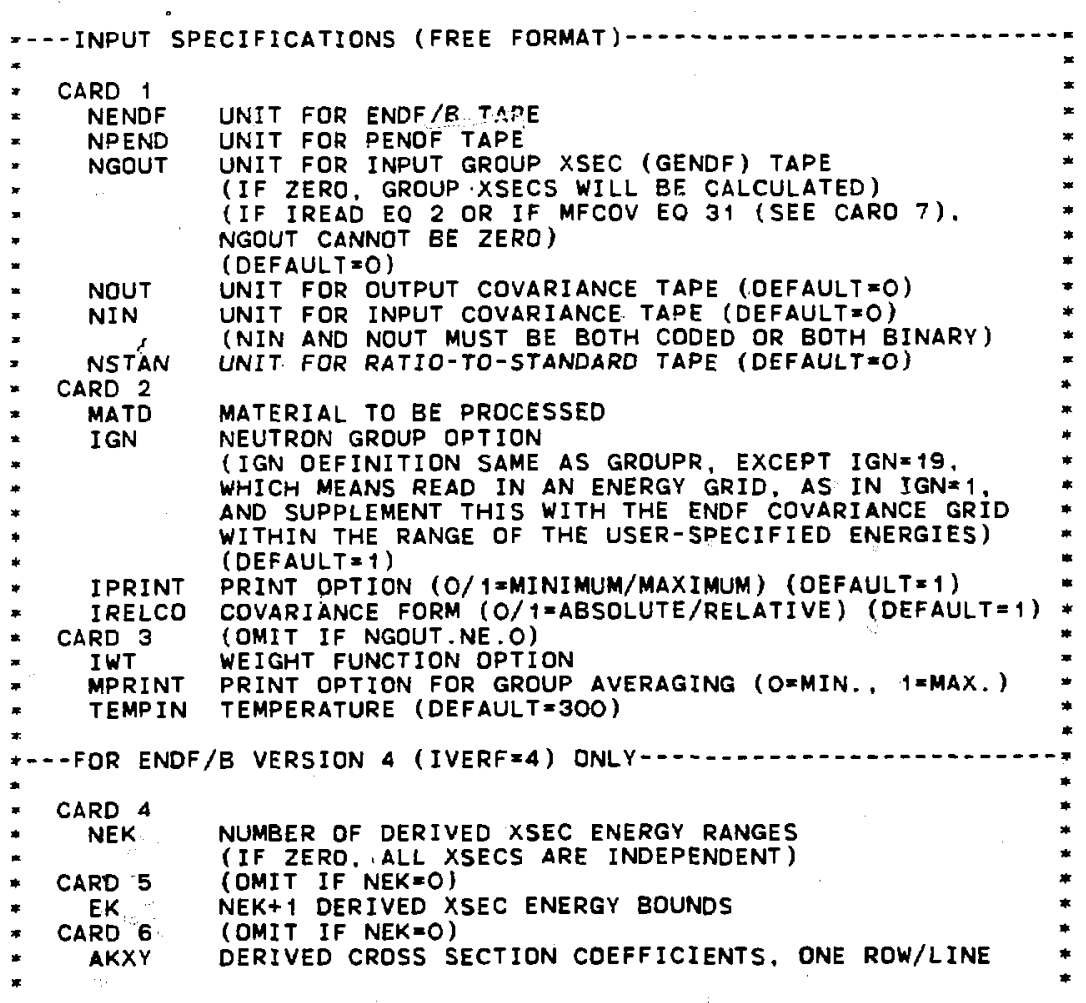




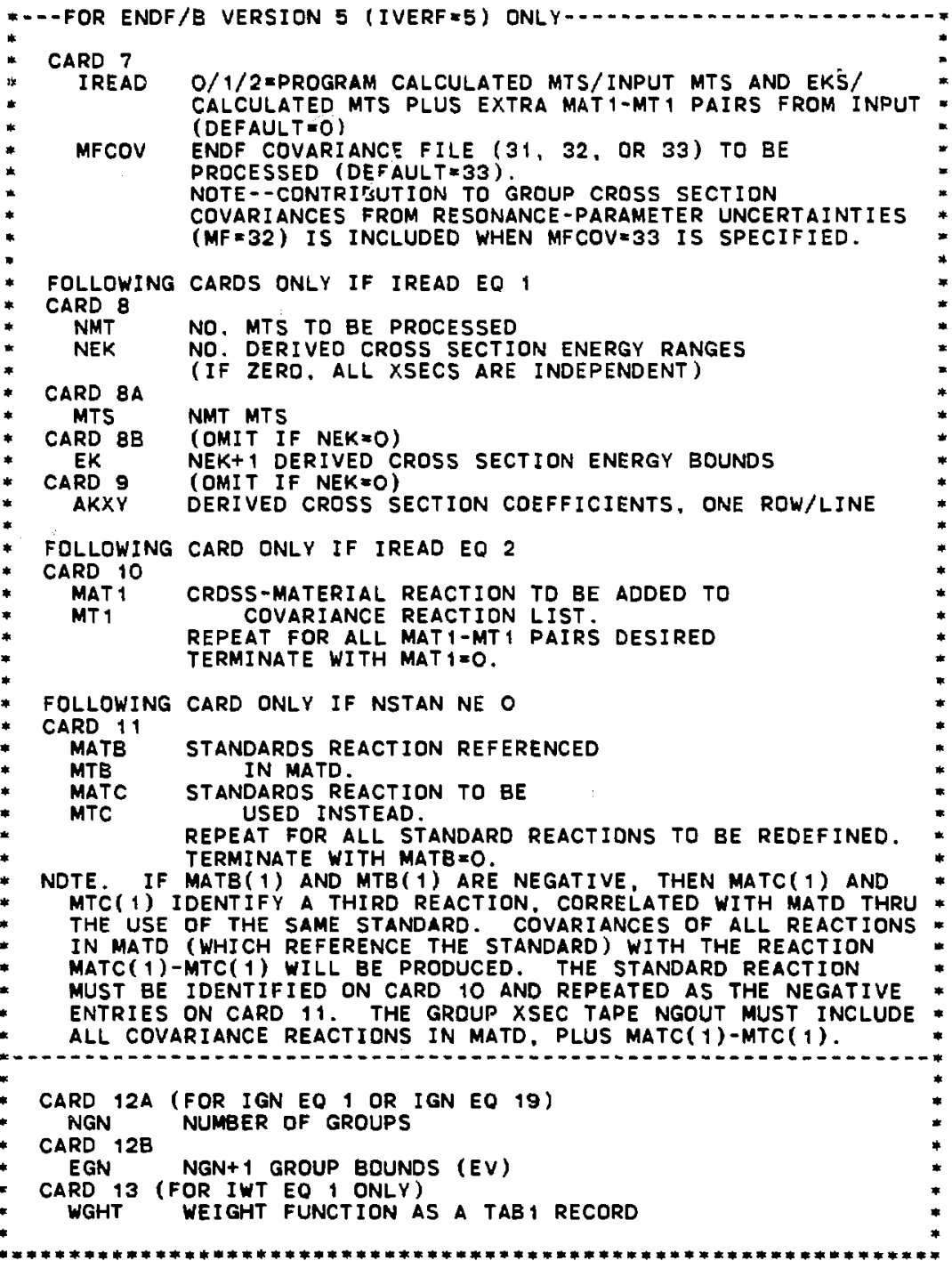


Further details on many of these input parameters are provided in the text below. Actual NJOY input for a few example problems is provided for further clarification. Complete input and output for yet another example problem are given in Ref. 5 .

NPEND, NGOUT... The user must supply either a PENDF tape on unit NPEND or a GENDF tape on unit NGOUT. If present, the PENDF tape should contain pointwise (that is, resonance-reconstructed, but not multigrouped) data and is normally produced with the RECONR module, ${ }^{6}$ although if the resonance region is of no interest, a copy of the ENDF will suffice. The GENDF tape, if present, should contain multigroup cross sections and/or $\bar{v}$ values, produced by the GROUPR module, for all reactions for which multigroup covariances are needed. Group cross sections need not be in the same group structure as the requested output covariances, although if the input group structure is much coarser than the output structure, rather crude approximations will be made in deriving an effective set of fine-group cross sections from the coarse input data.

NIN, NOUT... Input and output covariance tapes in the user's group structure; may be either both formatted or both binary. Although ERRORR lacks an explicit multimaterial loop, the same effect can be achieved by executing a series of ERRORR runs, with the output of one run becoming the input of the next. Only two unit numbers need be employed, with the data going back and forth between them until the multimaterial library is complete. To save time, binary files should be used for this purpose. MODER can be used to convert the final output tape from binary to formatted form, if desired.

NSTAN... This tape is needed if covariances are requested for a reaction pair that is related by ratio measurements. The ENDF subsection for such a pair will contain an NC-type sub-subsection with LTY $=1,2$, or 3 . The specific pairs in ENDF/B-V that require a standards tape are tabulated in Table $I$. For other evaluations, one can assume NSTAN is not needed. The code will stop with a clear error message if it actually is needed.

IREAD... For Version $v$, the list of reactions for which covariances are produced is constructed in one of several ways, depending on the value of IREAD. If IREAD $=0$, the reaction list is assumed to be identical to the list of sections contained in the ENDF/B covariance file (see MFCOV below). Although IREAD $=0$ is the most convenient and most commonly used option for constructing the list of covariance reactions, it leads to a problem if some covariance reactions have thresholds above the highest group boundary of the user's structure. These reactions will have zero cross sections in all groups and thus 
will be omitted from NGOUT. To avoid this difficulty, ERRORR automatically resets the user's highest group boundary to $20 \mathrm{MeV}$ whenever IREAD=0. By judicious choice of weighting functions, one can minimize the effect of this resetting on the other cross sections and their covariances.

If IREAD $=1$, only the "covariance" reactions specifically named by the user will be included in the reaction list. For certain applications, this option can save considerable execution time. However, to take advantage of this option, the user must examine the ENDF evaluation visually to determine which reactions are "derived" from which other reactions in each energy region. As discussed in Section $C$, this information is contained in NC-type sub-subsections with LTY $=0$. Incorrect results will be obtained if one requests processing for a given reaction without processing all of the reactions from which the given reaction is derived in the energy region spanned by the output group structure. The inclusion of those reactions may require, in turn, additional inclusions as well. In addition, the user must extract from the file (and enter into the input) the appropriate "derivation coefficients" and the energy ranges ovcr which they apply. An example of the required input is presented below in the discussion of the parameters NMT, NEK, MTS, EK and AKXY.

If IR $\mathrm{EAD}=0$ or 1 , a complete set of covariance matrices is output. That is, covariance matrices are produced for every reaction-pair combination that can be formed from the given reaction list. For those reaction pairs where the evaluator has not specified the covariances, the output matrix contains only zeros.

In an evaluation containing cross-naterial covariances, subsections involving the "other" materials will be ignored if IREAD=0 or 1 . These subsecţions can be selectively processed by specifying IREAD=2. If IREAD=2, the reaction list is initially constructed in the same way as for IREAD=0. This list is then supplemented with a list of extra reactions $\left(M A T 1_{k}, M T 1_{k}\right.$ ) (where MATl $_{k}$ FMATD), specified by the user. The output in this case contains matrices for all of the (MATD,MT ${ }_{i}$;MATD, MT ${ }_{j}$ ) combinations, as before, plus matrices for all (MATD, MT ${ }_{i} ; M A T 1_{k}, M T 1_{k}$ ) combinations. However, covariances among the extra reactions, for example: (MAT1 $1_{k}, M T 1_{k}$;MAT1 $1_{m}, M T 1_{m}$ ), are not computed. Input for an example problen that illustrates the use of IREAD $=2$ is given below, in the discussion of MFCOV. That discussion also includes an illustration (Table, II) of the set of MAT/MT combinations that are processed when IREAD $=2$. 
NFCOV... This parameter is used to specify whether covariances of fission $\bar{v} \quad(M F C O V=31)$ or cross sections (MFCOV=33) are needed. If $M F C O V=31$, it is necessary to supply multigrouped cross sections and $\bar{v}$ values on a GENDF tape (that is, NPEND must be 0); the sample problem below shows the calculational sequence required. MFCOV $=32$ is reserved for a planned future capability to compute uncertainties in self-shielded cross sections. If MFCOV $=33$, the contribution of the uncertainty in individual resonance parameters (File 32) is included in the calculated uncertainty in infinite-dilution group cross sections. Given below is the complete NJOY input for a calculation of a multigroup $\bar{v}$ covariance library for ${ }^{238_{\mathrm{U}}}$, including five cross-material reactions.

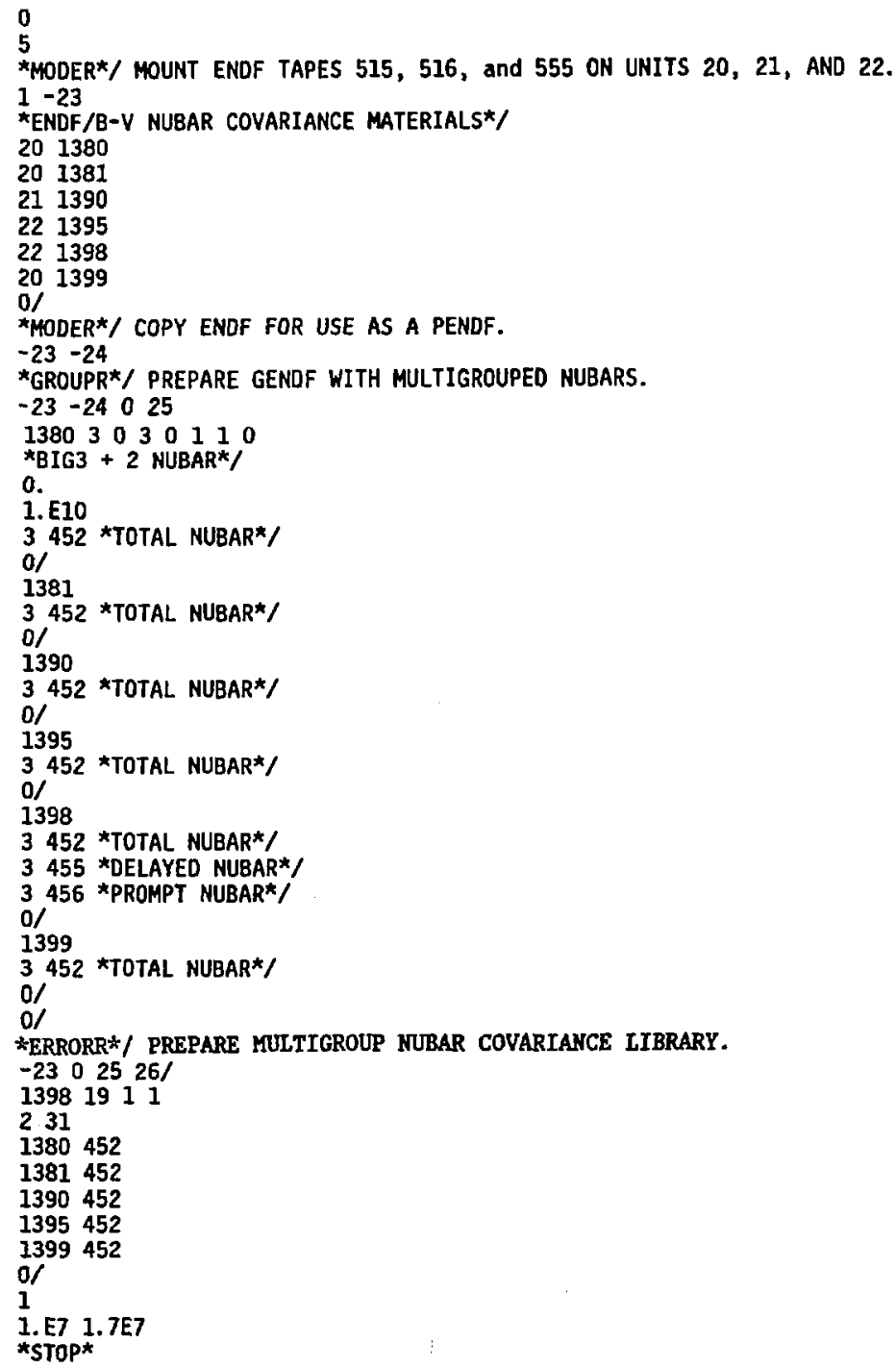


Note the use of the MODER module to prepare a special ENDF tape containing selected evaluations from other ENDF tapes. Because only high-energy $\bar{v}$ data are requested (10-17 MeV), it is unnecessary to use RECONR to prepare a PENDF tape for CROUPR. A copy of the ENDF tape is used instead.

Following the prescription given in the discussion of the IREAD $=2$ option, with this particular input covariance matrices will be produced for the reaction pairs marked with an $\mathrm{X}$ in the table below. The remaining non-redundant possibilities (dashes) can be filled in with successive ERRORR runs with MATD= 1380,1381 , etc.

TABLE II

REACTION-PAIRS IN ${ }^{238}$ U LIBRARY

\begin{tabular}{|c|c|c|c|c|c|c|c|c|c|}
\hline & $\mathbf{a}$ & $\mathbf{b}$ & c & d & e & $\mathbf{f}$ & 8 & $\mathbf{h}$ & $\mathbf{i}$ \\
\hline$a=(1398,452)$ & $x$ & $x$ & $x$ & $x$ & $\mathrm{x}$ & $x$ & $x$ & $x$ & $x$ \\
\hline$b=(1398,455)$ & & $x$ & $x$ & $\mathrm{X}$ & $\mathrm{x}$ & $\mathrm{X}$ & $\mathrm{x}$ & $\mathrm{x}$ & $\mathrm{x}$ \\
\hline$c=(1398,456)$ & & & $x$ & $\mathrm{X}$ & $\mathrm{X}$ & $\mathrm{X}$ & $x$ & $\mathrm{x}$ & $x$ \\
\hline$d=(1380,452)$ & & & & - & - & - & - & - & - \\
\hline$e=(1381,452)$ & & & & & - & - & - & - & - \\
\hline$f=(1390,452)$ & & & & & & - & - & - & - \\
\hline$g=(1395,452)$ & & & & & & & - & - & - \\
\hline$h=(1398,452)$ & & & & & & & & - & - \\
\hline$i=(1399,452)$ & & & & & & & & & - \\
\hline
\end{tabular}

MMT, NEK, MTS, EK, AKXY... For Version V, if IREAD=1, the user specifies a subset of the evaluator's "covariance reactions" (that is, a subset of the sections of MFCOV), as the particular set of reactions for which processing is requested. These NMT desired reactions are entered in the array MTS. As mentioned in the discussion of IREAD above, one must also include in the MTS array all reactions from which the desired reactions are "derived."

Another requirement of the IREAD $=1$ option is that the actual derivation coefficients (called AKXY in the code, but called $C_{I}(a, c)$ in the discussion in Section G) must be entered in the ERRORR input for NEK energy ranges spanning the output group structure. Adjacent ENDF/B derivation ranges should be merged 
into a single range if the derivation-coefficient matrix for the NMT explicitly requested reactions is the same in each of the adjacent ranges. The ordering of the $C_{I}(a, c)$ data is as follows: on one line of input the coefficients are specified for a fixed a-value and for all c-values ranging from 1 to NMT. One such line is given for each a-value. Finally, there is an outer loop over the NEK energy ranges. An example of the input that is required for IREAD $=1$ is given below for the case of ENDF/B-V carbon MAT1306. It will be necessary to examine File 33 of the evaluation (which is available on the ENDF/B-V standards file, Tape 511), in order to understand the details of this example.

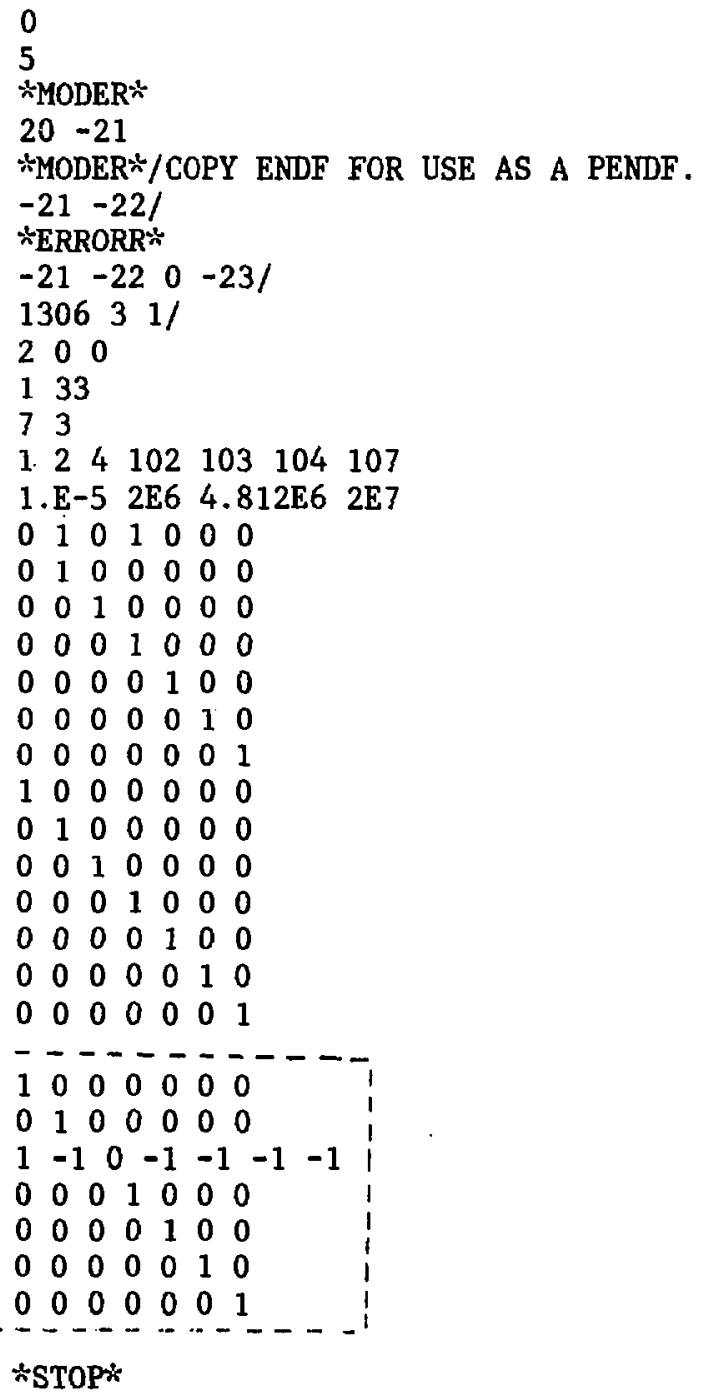


Note that here, even if the user wanted to process only MT2 and MT4, for example, it is nevertheless necessary to include MT1, MT102, MT103, MT104, and MT107, because the $I G N=3$ group structure extends to $17.0 \mathrm{MeV}$, and above 4.812 $\mathrm{MeV}, \mathrm{MT} 4$ is derived from the relation

$$
\sigma_{4}=\sigma_{1}-\sigma_{2}-\sigma_{102}-\sigma_{103}-\sigma_{104}-\sigma_{107}
$$

See the corresponding $C_{I}(a, c)$ matrix in the input above (dashed box).

MATB, MTB, MATC, MTC... Card 11 provides a facility for, in effect, going into the covariance file and changing all references to a given standards reaction (MATB,MTB) appearing in NC-type sub-subsections with LTY $=1,2$, or 3 into references to a different reaction (MATC,MTC). (See the discussion of ratio measurements in Section $H_{\text {.) }}$ This facility is useful if the evaluation (MATB, MTB) is for some reason unavailable. Up to 5 such standards can be redefined. As explained in the note on the input instructions at the beginning of this section, Card 11 is also used (if MATB(1) and MTB(1) are negative) to process covariances between two distinct reactions, both measured relative to a common standards reaction. This was referred to as the "LTY $=4$ " case in Section H. A separate ERRORR run is required for each requested "LTY $=4$ " reaction pair.

\section{ERRORR Output File Specification}

The results from ERRORR are written to an output file on unit NOUT, provided that the input parameter NOUT is non-zero. This file contains the user's group structure, the multigroup cross sections, and either absolute (IRELCO=0) or relative (IRELCO $=1$ ) covariances. If NOUT is positive, then a formatted (card-image) file is written, and, if uegative, a binary file is written. In either case, the output is written with the standard NJOY I/O utilities, ${ }^{5}$ and the result has an ENDF-like structure. As is the case for ENDF, PENDF, and GENDF files, ERRORR output files can be converted from binary to formatted form (and vice versa) by using the MODER module. The energy-group ordering of the data written to NOUT follows the ENDF convention. That is, low-energy groups precede the high-energy groups.

A small, stand-alone retrieval progran, which reads formatted ERRORR output files, reorders the energy groups to high-to-low order, and fills in the implicit zeroes to make a full matrix, is listed as Table IV in Ref. 11. A much more powerful retrieval program, which produces libraries for sensitivity 
codes and produces publication-quality plots of the data, starting from either binary or formatted ERRORR output, is the COVR module of NJOY. This module is described in the following chapter of this report.

It is occasionally of interest to examine a formatted ERRORR output file visually. For this and other purposes, we describe below the detailed form of the formatted output file. To assist in the description, we give in Table III an example of such a file.

The data in Table III are written in the form of standard, 80-column card-images consisting of 10 data fields. The first 6 fields are 11 characters wide and are used for either floating-point numbers or integers. The seventh, eighth, ninth, and tenth fields are 4, 2, 3, and 5 characters wide, respectively, and are used for integers only. The four digits in Field 7 are the MAT, or material, number of the isotope or element; the two digits in Field 8 are the MF, or file, number that indicates the general type of data (for example, cross-section data vs covariance data); the three digits in Field 9 are the MT, or section, number that indicates the particular nuclear reaction; and finally, the five digits in Field 10 are the card sequence number. Sections are terminated by zeroes in the MT field, files by zeroes in the MF field, and materials by zeroes in the MAT field.

The first card shown in Table III is the first card for the material MAT 1326, which is elemental iron. Note that on this card the number $2.6 \times 10^{4}$ appears in the first field and 55.365 is the second field. These are the "ZA" $(1000 \times 2+A)$ and "AWR" (atomic weight relative to the neutron), numbers taken directly from the ENDF/B file. The fact that $Z A$ is 26000 (suggesting that $A=$ 0) is a convention that means that the data are for the element $F e$, rather than for a single isotope.

Also note that $\mathrm{MF}=1$ and $\mathrm{MT}=451$ on cards 1 to 8 . This MF-MT combination is used in ENDF/B for descriptive Hollerith information, but it is used here for the boundaries of the multigroup structure used for the processed data to follow. On card 2, note the number 30 in Field 3 and the number 31 in Field 5, which indicate, respectively, the number of energy groups and the number of energy-group boundaries in the multigroup set. The values of the group boundaries, given in $\mathrm{eV}$ from low to high energy, follow on cards 3 through 8 . Cards 9 and 10 are section and file terminators, respectively.

Multigroup cross-section data are given in barns on cards 11 through 101. This file, denoted by $M F=3$, corresponds to the smooth cross-section file in 
ENDF/B. The MT-numbers (Field 9) can be identified with particular nuclear reactions by consulting Appendix B of Ref. 5.

\section{TABLE III}

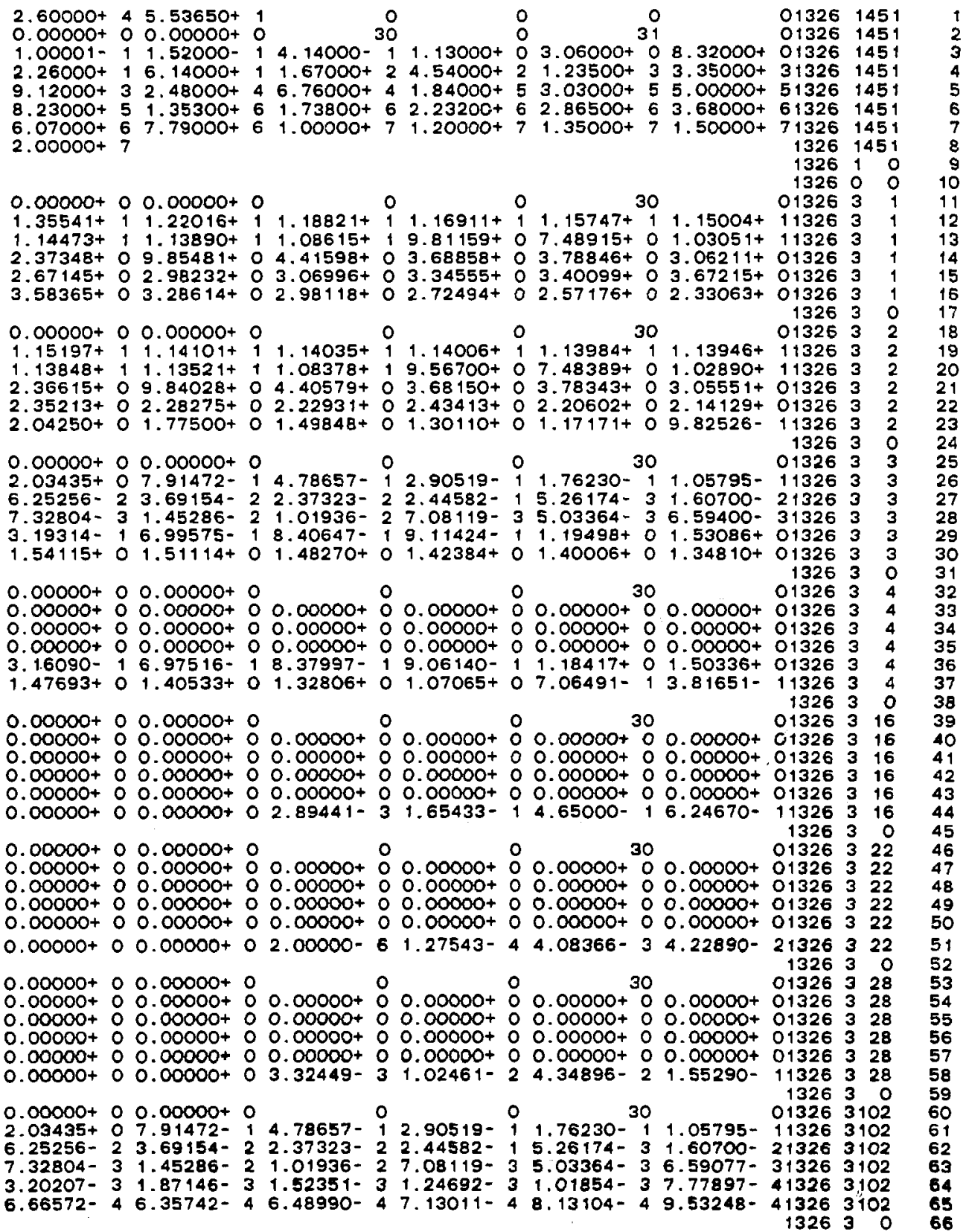




\section{TABLE III (Cont.)}

$0.00000+00.00000+0$

30

013263103

$0.00000+00.00000+00.00000+0.0 .00000+00.00000+00.00000+013263103$

$0.00000+00.00000+00.00000+00.00000+00.00000+00.00000+013263103$

$0.00000+00.00000+00.00000+00.00000+00.00000+03.23000-613263103$

$2.22100-51.87870-41.12710-34.03744-39.77153-32.60238-213263103$

$5.64685-28.63289-21.13654-11.28663-11.20209-17.33574-213263103$ $0.00000+00.00000+0 \quad 0 \quad 0 \quad 0 \quad 30 \quad 013263104$

$0.00000+0.0 .00000+0.0 .00000+0.0 .00000+00.00000+00.00000+013263104$ $0.00000+00.00000+00.00000+00.00000+00.00000+00.00000+013263104$ $0.00000+0.0 .00000+00.00000+00.00000+00.00000+0.0 .00000+013263104$ $0.00000+00.00000+00.00000+00.00000+00.00000+00.00000+013263104$ $0.00000+01.80996-52.25000-39.13333-31.99333-22.86600-213263104$

$0.00000+00.00000+0 \quad 0010013263105$ $0.00000+00.00000+00.00000+0.0 .00000+00.00000+00.00000+013263105$ $0.00000+0.0 .00000+00.00000+0.0 .00000+00.00000+00.00000+013263105$ $0.00000+00.00000+00.00000+00.00000+00.00000+0.0 .00000+013263105$ $0.00000+00.00000+00.00000+00.00000+00.00000+00.00000+013263105$ $0.00000+00.00000+00.00000+00.00000+05.60000-41.44000-213263105$ 132630

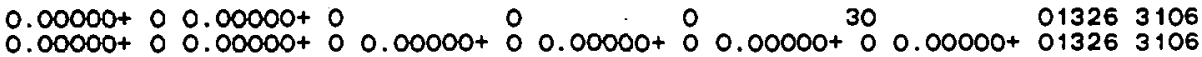

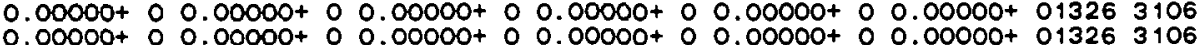
$0.00000+0.0 .00000+00.00000+00.00000+00.00000+00.00000+013263106$ $0.00000+00.00000+00.00000+00.00000+00.00000+00.00000+013263106$ $0.00000+00.00000+00.00000+08.33334-63.91667-45.31000-313263106$

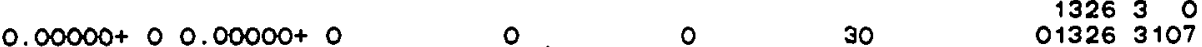
$0.00000+00.00000+00.00000+0.0 .00000+0.0 .00000+00.00000+013263107$ $0.00000+00.00000+00.00000+00.00000+00.00000+00.00000+013263107$ $0.00000+00.00000+00.00000+00.00000+0.0 .00000+00.00000+013263107$ $0.00000+00.00000+00.00000+00.00000+01.98577-56.90973-413263107$ $7.08327-31.88243-23.18674-23.88640-23.90835-22.15230-213263107$ 132630 $\begin{array}{llll}1326 & 0 & 0 & 102\end{array}$

$2.60000+45.53650+1$ $0.00000+00.00000+0$ $0.00000+00.00000+0$ 2. $11317-32.11788-3$

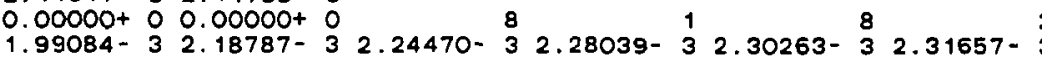
$2.32507-32.33026-3$

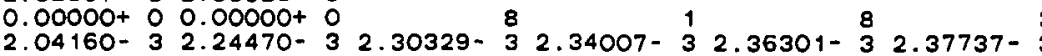
$2.38619-32.39151-3$ $0.00000+00.00000+0$ $2.07346-32.28039-3$ $2.42456-32.42997-3$ $0.00000+00.00000+.0$ $2.09334-32.30263-3$ $2.44849-32.45396-3$ $0.00000+0.0 .00000+0$ $2.10578-32.31657-3$ $2.46347-32.46897-3$ $0.00000+00.00000+0$ $2.11317-32.32507-3$ $2.47284-32.47833-3$ $0.00000+00.00000+0$ $2.11788-32.33026-32.39151-32.42997-3,45396-3 \quad 8.46897-3132633$ $2.47833-32.48385-3$ $0.00000+0.00000+0$ $0.00000+00.00000+0 \quad 9096132633$ $5.04370-8 \quad 4.19464-8 \quad 1.45226-8$ $0.00000+00.00000+0$ 909010132633 $9.78388-51.01291-41.75139-73.88732-77.69641-73.67503-7132633$ $5.75422-74.78555-71.65684-7$ $0.00000+0.0 .00000+0$ $1.53513-8 \quad 1.75139-79.98645-59.97849-59.96429-51.03579-8132633$ $1.62180-81.34879-84.66973-9$ $0.00000+00.00000+0$ 9

30132633

1132633 132633 2132633 132633 3132633 132633 4132633 132633 5132633 132633 6132633 132633 7132633 132633 132633
132633 8132633$$
\text { • }
$$ 


\section{TABLE III (Cont.)}

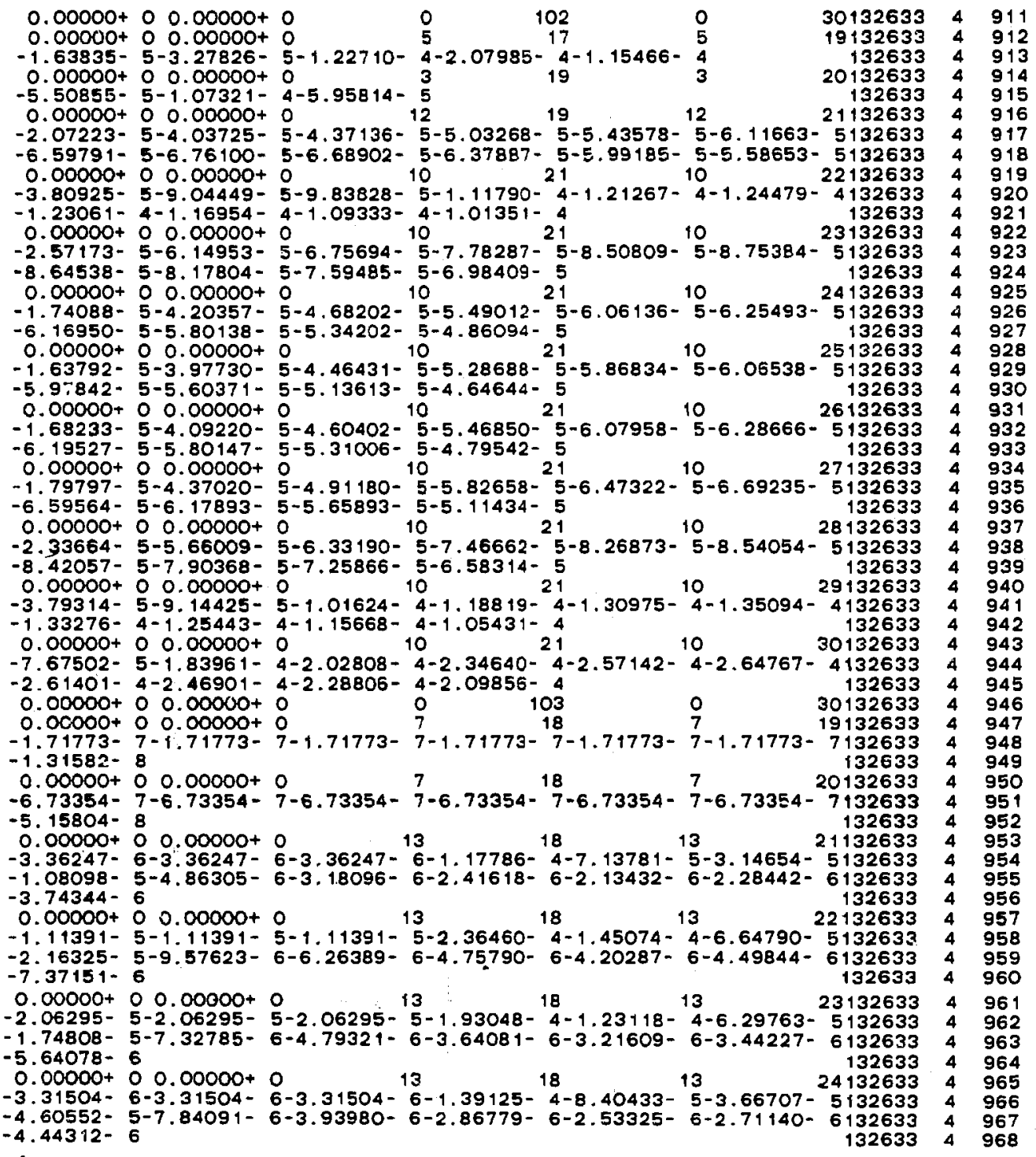

The multigroup covariance data for MAT1326 (Fe) begin with card 103 . The designation of MF $=33$ in Field 8 is the same as that for covariance data in ENDF/B. Card 103 repeats the ZA and AWR numbers in Fields 1 and 2, and the number 13 in Field 6 indicates that covariance matrices for 13 reaction-pairs follow. The number " 1 " in the MT field (Field 9) indicates that all 13 reaction-pairs have $M T=1$ as the "first" reaction. 
The data for one typical zeaction-pair begin at card 911 . In this section, note that the "first" reaction is MT $=4$. Field 4 of card 911 contains the number "102," so the "second" reaction is MT1 $=102$. In other words, the data to follow refer to the covariance matrix of the iron inelastic scattering reaction (4) with the iron radiative capture reaction (102). The number "21" occurring in Field 6 of Card 916 indicates that these data refer to Group 21 of $\mathrm{MT}=4$, i.e., the twenty-first row of the covariance matrix. The number " 12 " in Field 3 of Card 916 indicates that there are 12 consecutive groups of $\operatorname{Mr} 1=102$ for which covariances are given explicitly. The first covariance applies to group 19 of MT1 $=102$, as indicated by the number " 19 " in Field 4 . Then, in referring to the entry in the sixth field of Card 917, for example, one would say that "the relative covariance of the iron inelastic cross section in Group $21(1.738-2.232 \mathrm{MeV})$ with the iron radiative capture cross section in Group 24 $(3.68-6.07 \mathrm{MeV})$ is $-6.11663 \times 10^{-5} . "$

Any row of a covariance matrix, or any part of a row, that contains only zeroes will be omitted from the ERRORR output. The only exception to this rule is that the last row (Group 30 in this example) is always given explicitly, even if it does contain only zeroes. The occurrence of the last row terminates a given covariance matrix and signals that the next card will name a new reaction-pair.

Although the output file in this example contained no cross-material covariances, the only difference in the file structure in the cross-material case is that MAT1 is specified in the integer field just preceding MT1, for example, in Field 3 on card 911. The entry MAT1 $=0$ in that position here is a flag to indicate that $\mathrm{MAT} 1=\mathrm{MAT}=1326$.

\section{Error Messages}

ERRORR * + XILLEGAL IREAD = ---

Currently defined values are 0,1 , and 2 .

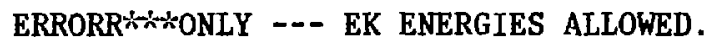

The number of derived-cross-section energy-range boundaries is limited to 50 .

ERRORR나서T MANY REACTION TYPES.

This error occurs in runs with IVERF $=4$ if the number of requested covariance reactions is more than 60 . A possible solution is to use the 
IREAD = 1 option to select only the most needed reactions. This error also occurs in runs where internal group averaging is requested (NGOUT = 0) if there are more than 200 sections in $M F=33$. An alternative is to run GROUPR first, then ERRORR with NGOUT.GT.0.

ERRORR $\rightarrow$ H $\%$ NOT CODED FOR MFCOV $=---$.

Allowed values are 31 and 33 .

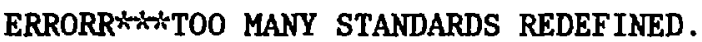

Limit is 5 . See input for NSTAN.NE.0.

ERRORR* $*$ ILLEGAL MT GT 870.

Fault in evaluation.

GRID APPEAR IN MFCOV.

Fault in evaluation or input.

GRID

Limit is 60 .

GRID $\stackrel{2}{*}$ KCANNOT CALCULATE COVARIANCES OF REACTION MT = --- WITH MT1 = --- BECAUSE NSTAN $=0$. TO PROCEED, MOUNT AN ENDF TAPE CONTAINING THE' STANDARD REACTION (MATSTD $=---$, MTSTD $=---$ ) ON UNIT NSTAN. IF NECESSARY MATSTD AND MTSTD CAN BE REDEFINED ON INPUT CARD 11.

Self-explanatory.

GRID $2+x$ COVARIANCES OF REACTION MT $=--$ WITH (MAT1 $=--$, MT1 = --) CANNOT BE CALCULATED. NOT CODED FOR LTY $=\cdots$.

Fault in evaluation.

GRID $+\frac{1}{x+1}$ TOO MANY REACTION TYPES.

This error occurs if IVERF $=5$ and if the requested number of covariance reactions is greater than 60 . If cross-material covariances are not needed, then a possible solution is to use the IREAD = 1 option to select only the most needed reactions.

GRID

Fault in evaluation. Evaluator may have meant to indicate MT1 $=M T$. If so, change the data value for MT1 on NENDF from 0 to MT and resubmit. MERGE $x+$ STORAGE EXCEEDED.

Either the number of points in the $x$ grid exceeds 150 or the number in the y grid exceeds 1000 . 


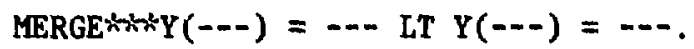

Should not occur.

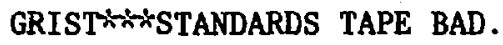

Fault in evaluation. Standards reactions must have at least one subsection; that is, they may not be components of a lumped reaction.

GRISTWHALILLEGAL LB $=0$.

Fault in evaluation. Absolute covariances are not permitted in the standards.

GRIST每-

Fault in evaluation. Standards reactions may not be derived from other reactions.

LUMPMT $\leftrightarrow$ 년.

The number of component reactions of a lumped reaction is limited to 50 . COVCAL int STORAGE EXCEEDED IN LOC.

The number of NI-type sub-subsections in any subsection is limited to 30 . COVCAL

Fault in evaluation. LB-LT combination is not defined. COVCAL $\div$ NOT CODED FOR LB $=-\cdots$.

Currently defined values are 0 through 6.

- COVCAL" $\pitchfork$ STORAGE EXCEEDED IN EGT.

At the time of COVCAL processing, the number of groups on NGOUT (equal to the number of union groups) is limited to 1000 .

COVCAL $\because-\mathrm{K}_{\mathrm{K}} \mathrm{MCOV}$ MT FOUND NOT EQUAL TO INPUT MT.

Conflict between internal list of covariance reactions and the reactions encountered in ENDF file. Should not occur.

COVCAL $\approx$ STORAGE EXCEEDED IN A.

Insufficient space in core to store the information from NI-type sub-subsections. Increase the size of /ESTORE/ and NAMAX.

COVCAL $\%$ ILLLEAL MT1 $=0$.

See similar diagnostic in SUBROUTINE GRID above. 
COVCALฬฬ*DATA IN A(LOCI) ARE ILLEGAL.

Fault in evaluation. Bad ratio-to-standard data. Either LTY is outside the

legal ENDF range of 1-3 or the energies EL, EH are negative or out of order.

COVAL * *MUST REQUEST MAT1 $=\ldots$ AND MT1 $=\ldots$ ON CARD 10.

Input error in requesting covariances of $X$ with $Y$ when both are measured relative to $Z$. The standard $Z$ must be specified on Card 10 and as negative entries on Card 11.

ROGOUT*

Cannot find MAT on multigroup library.

RDGOUT*2**MF---, MT--- NOT FOUND.

Cannot find requested group structure or cross sections on multigroup library.

RDGOUT\%ฬฬBAD INDEX FOR B EQUIVALENT TO SIG(IG).

Should not occur.

COVOUTHस:HNABLE TO FIND IY OR IYP FROM MTS ARRAY.

Conflict between internal list of covariance reactions and the reactions encountered on the union-group covariance file produced in CovCAL. Should not occur.

COVOUT* $*$ STORAGE EXCEEDED IN SUM.

Insufficient space in core to store the NMT1 ${ }^{\text {NNGN }}$ user-group cross sections. Increase the size of /ESTORE/ and NAMAX.

COVOUT $* * *$ UNEXPECTEDLY, IX NE IY OR IXP NE IYP.

Should not occur.

SIGC**\%OOVARIANCE REACTION MISSING FROM LUMPING TABLE.

A covariance reaction in the 851-870 range cannot be found in the lumped reaction definition tables. Should not occur.

RESPRP $*$ ILLEGAL RESONANCE REPRESENTATION IN MF $=32 . \quad$ LRF $=-\cdots$.

Fault in evaluation. Allowed values of LRF are 1 and 2 (single- and multi-level Breit-Wigner representations).

RESPRP***STORAGE EXCEEDED.

Insufficient space in array ISCR to store resonance-parameter covariances. for current L-value. Limit is 5006 words. 
RESPRP $r+\div$ BAD COVARIANCE DATA FOR RESONANCE PARAMETERS --- AND -- AT ER = - -

Fault in evaluation. Covariance matrix of the parameters has either negative variances, or infinite correlation coefficients, or correlation coefficients greater than 2.0. Sata may be out of order.

GRPAV--MF -.. MT -.. HAS THRESHOLD GT HIGHEST UNION ENERGY.

Though not a fatal error, this message often precedes an abort in COVCAL caused by the absence of this reaction on NGOUT. If IREAD $=1$, then delete this reaction from the requested list of covariance reactions.

GRPAV*rituNABLE TO FIND TEMP = ---.

Cannot locate requested material and temperature on NPEND.

GRPAV:-iCANNOT GROUP-AVERAGE MT =---. USE GROUPR FIRST, THEN ERRORR WITH NGOUT . NE . 0 .

The MFCOV $=31$ and NGOUT $=0$ options are not compatible.

GRPAV:-nNNT CODED FOR MULTIMATERIAL GROUP AVERAGING. USE GROUPR FIRST, THEN ERRORR WITH NGOUT NE 0 .

The IREAD $=2$ and NGOUT $=0$ options are not compatible.

COLAPS $* \because-\div D I D$ NOT FIND EXPECTED MF1 MT451.

Multigroup libraries produced with the GROUPR modules always contain the group structure in $\mathrm{MF}=1, \mathrm{MT}=451$.

COLAPS

Number of groups in NGOUT "library" structure is limited to $2 *$ NPAGE+50, which, for the standard value of NPAGE (306), gives a limit of 662 groups.

COLAPS

The supplied library must cover the entire energy range of the union grid, which is the same as the energy range of the user's grid.

COLAPS

Because of this restriction, existing "general-purpose" GENDF files may not be suitable for use as input to ERRORR, in which case a special GROUPR run, just to prepare the NGOUT file for ERRORR, will be required.

UNIONG

Applies only if IVERF $=4$.

UNIONG

Applies only if IVERF $=4$. 
UNIONGW

Insufficient space in core to store the union grid. Increase/ESTORE/ and NAMAX.

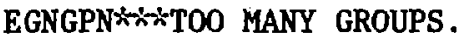

User's group structure is limited to 640 groups.

EGNGPN:

With the IGN = 1 option, the supplied group structure must be in monotonically increasing order.

EGNGPN负浞ILLEGAL GROUP STRUCTURE REQUESTED.

Allowed values of IGN are 1-15 and 19 .

EGNWTFWHILLEGAL WEIGHT FUNCTION REQUESTED.

Allowed values of IWT are 1-9.

EGTSIG

In the NGOUT $=0$ option, a point cross section has been requested by GRPAV for an illegal MT-number. In this context, the allowed values of MT are 1-150, 207, 251-253, and 600-799. MT-numbers 600-699 are not defined in ENDF/B- $V$, but may be used on ENDF and PENDF files specially prepared as input to ERRORR, to allow processing of nonstandard reaction types.

N. Input/Output Units

The following logical units are used.

11 NSCR in COVCAL and COVOUT. Contains the union-group multigroup covariances for the directly evaluated reactions.

12 NSCR2 in COVOUT. A second copy of NSCR, which is read only for the "trivial" derivation case, ISD $=1$. Saves execution time by eliminating the calculation of wany zero contributions.

13 NSCRG in COVCAL and RDGOUT. Used to extract a single MAT from a multimaterial NGOUT.

15 NSCR in LUMPTMT and LUMPXS. Used if MT-numbers in the 851-870 range (lumped reactions) are found in ENDF covariance file to keep a copy of the covariance file.

20-99 Users' choice for NENDF, NPEND, NGOUT, NOUT, NIN, and NSTAN.

Units 11 and 12 are normally binary, although they can be switched to formatted mode by changing the value of the variable IMODE in the main progran to +1 . 
Unit 13 has the same mode as NGOUT. The user can choose the modes for NENDF, NPEND, NGOUT, NOUT, NIN, and NSTAN, except that NOUT and NIN must have the same mode.

\section{Acknowledgments}

Much of the programming of the ERRORR and COVR (see following chapter) modules was performed by R. M. Boicourt. We offer thanks to D. J. Dudziak for his sustained interest in our development of covariance methods and libraries, to R. J. LaBauve for his work on the initial processing of many of the ENDF/B-V evaluations, to $\mathrm{C}$. Stenberg for his assistance in the development of IBM versions of the two modules, and to R. W. Peelle and B. H. Broadhead for helpful advice in several areas. Thanks are also due for the encouragement received from the Office of Fusion Energy. Partial financial support for the preparation of this report was provided in the sumer of 1985 , by the Swiss Federal Institute for Reactor Research (EIR) at Würenlingen, where one of the authors (D. W. Muir) served as a guest scientist.

\section{P. References for ERRORR}

1. S. A. W. Gerst1, D. J. Dudziak, and D. W. Muir, "Cross Section Sensitivity and Uncertainty Analysis with Application to a Fusion Reactor," Nucl. Sci. Eng. 62, 137 (January 1977).

2. W. A. Reupke, D. W. Muir, and J. N. Davidson, "Consistency of Neutron Cross-Section Data, S Calculations, and Measured Tritium Production for a 14-MeV Neutron-Driven Sphere of Natural-Lithium Deuteride," Nucl. Sci. Eng. 82, 416 (December 1982).

3. W. C. Hamilton, Statistics in Physical Science (Ronald Press, New York, 1964). Section 4-8 gives a compact proof of the Gauss-Markov theorem. As applied to the problem of updating a cross-section evaluation, this theorem states that the generalized method of least squares provides the best possible estimates (compared with other linear combinations of the input data) of both the cross sections and all linear functions of the cross sections.

4. R. Kinsey, Ed., "ENDF-102, Data Formazs and Procedures for the Evaluated Nuclear Data File, ENDF/B-V," Brookhaven National Laboratory report BNLNCS-50496 (ENDF-102) (revised by B. A. Magurno, November 1983). (See especially Chapters 30-33.)

5. R. E. MacFarlane, D. W. Muir, and R. M. Boicourt, "The NJOY Nuclear Data Processing System, Vol. I: User's Manual," Los Alamos National Laboratory report LA-9303-M (ENDF 324) (May 1982). 
6. R. E. MacFarlane, D. W. Muir, and R. M. Boicourt, "The NJOY Nuclear Data Processing Sytem, Vol. II: The NĩY, RECONR, BROADR, HEATR, and THERMR Modules," Los Alamos National Laboratory report LA-9303-M (ENDF 324) (May 1982).

7. C. R. Weisbin, E. M. Oblow, J. Ching, J. E. White, R. Q. Wright, and J. Drischler, "Cross Section and Method Uncertainties: The Application of Sensitivity Analysis to Study Their Relationship in Radiation Transport Benchmark Problems," Oak Ridge National Laboratory report ORNL-TM-4847 (ENDF-218) (August 1975). (See especially Chapter IV.)

8. J. D. Smith III, "Processing ENDF/B-V Uncertainty Data into Multigroup Covariance Matrices," Oak Ridge National Laboratory report ORNL/TM-7221 (ENDF-295) (June 1980).

9. D. W. Muir, "Format Modification FM81-1 for Lumped Reaction Covariances," Attachment 5B2 to a memorandum from S. Pearlstein to Participants of the Cross Section Evaluation Working Group (CSEWG) on the subject "Summary of the CSEWG Meeting, 5/13-15/81," July 1, 1981.

10. P. G. Young, "Variance-Covariance Analysis of $n+{ }^{7} \mathrm{Li}$ Reactions," in Los Alamos National Laboratory report LA-8874-PR, pp. 2-10 (July 1981).

11. D. W. Muir and R. J. LaBauve, "COVFILS: A 30-Group Covariance Library Based on ENDF/B-V," Los Alamos National Laboratory report LA-8733-MS (ENDF-306) (March 1981). 
$\mathrm{XX} . \quad$ COVR

\section{A. Introduction}

The COVR module of NJOY is an editing module that post-processes the output of ERRORR in a manner analogous to the way MMATXS and DTFR post-process the output of GROUPR. COVR performs two quite separate functions, using the multigroup covariance file from ERRORR as input. First, it can prepare a new covariance library in a highly compressed card-image format, which is suitable for use as input to sensitivity analysis programs. ${ }^{1,2}$ (Data in this form can also be copied to the system OUTPUT file to obtain a compact printed summary of an ERRORR run, without using the sometimes bulky long-print option in ERRORR. Such a summary can provide information on standard deviations and correlation coefficients, neither of which are printed by ERRORR.) The second function of COVR is to produce publication-quality plots ${ }^{3}$ of the multigroup covariance information. We first describe the "library" option.

\section{B. Production of Boxer-Format Libraries}

As discussed in Section L of the ERRORR manual, Chapter XIX of this report, the output file of that module contains the group structure, cross sections, and either absolute (IRELCO $=0$ ) or relative (IRELCO $=1$ ) covariances for one or more materials, in either card-image or NJOY blocked-binary form. If NOUT is greater than zero, COVR reads an ERRORR output file from unit NIN and produces a new multigroup covariance library on unit NOUT. COVR performs only sorting and reformatting operations on the ERRORR data.

In COVR, as well as ERRORR, the ENDF/B energy ordering is followed. That is, low group indices correspond to low energies, high indices to high energies. Regarding group structures, in the library mode of operation, COVR (like ERRORR) makes extensive use of disk storage, so that even on the CDC 7600 , which has a relatively small central memory, very large group structures (up to 640 groups) can be handled successfully. (See, however, the comments at the end of this section regarding group-number limitations in the plot mode.)

The material and reaction coverage of the COVR output is determined by a set of reaction pairs (MAT,MT;MAT1,MT1) supplied by the code user (see input Card 4 in the input instructions and the corresponding discussion that follows in Section $D$ ). At the beginning of the output library, the group structure is given. Then, for each specified reaction pair, the output library contains either a covariance matrix or a correlation matrix, depending on the output 
option (MATYPE) selected. In the case that covariances are requested (MATYPE = 3 ), the type of data on NOUT (absolute vs relative) is governed by the covariance type present on NIN. In addition, whenever MAT1 = MAT and MT1 = MT, the group-cross-section vector and the (absolute or relative) standard-deviation vector for that particular reaction are written to NOUT just before the matrix itself. All data (group structure, cross sections, standard deviations, and matrices) are written to NOUT using a highly compressed, card-image format.

The design of this format, called the "Boxer" format, proceeds from a simple fact: as discussed in Chapter XIX, the ENDF/B-V covariance evaluations define certain rectangular regions (boxes) in energy "space," over which the relative covariance is constant. (The ENDF/B format allowing a constant absolute covariance is only rarely used.) The coordinate axes of the two-dimensional energy space in question are $E_{x}$ and $E_{y}$, where $x$ and $y$ indicate the particular reaction pair to which the ENDF/B covariances apply. Because of this feature of the basic evaluations, one expects that an element of a multigroup relative covariance matrix, derived from the ENDF/B data for a given reaction pair, frequently will be identical either to the element before it in the same row ( $E_{x}$ constant, $E_{y}$ varying), or to the element above it in the same column ( $E_{y}$ constant, $E_{x}$ varying). Thus, the Boxer format allows a combination of "horizontal" and "vertical" repeat operations.

Even though the ERRORR output format suppresses zero covariances, very large data compression factors can be achieved in transforming from the ERRORR format to the Boxer format. As one example, the ERRORR output file for a particular 137-group reactor-dosimetry library ${ }^{4}$ contained 38000 card images, while the corresponding COVR output file contained fewer than 1000 card images.

In the Boxer format, data are stored as a list of numerical data values (for example, relative covariances), together with a list of integers that control the loading of the data into the reconstructed array $c(i, j)$. A negative integer, say $-n$, indicates that the next value in the data list is to be loaded into the next $n j$-values of $C(i, j)$. A positive integer m means that, for the next $n$ j-values, the value to be loaded is simply carried down from the row above,

$$
c(i, j)=c(i-1, j)
$$

For the first row ( $i=1)$, the row "above" is defined to be a row containing all zeroes. 
In constructing the compressed data set in SUBROUTINE PRESS, the choice between using the "repeat-new-value" method or the "carry-down" method is made dynamically on the basis of taking the longest possible "step." If $m=n$, the "carry-down" method is chosen, as it does not require an entry in the data list. As an additional compression feature of the format, one may indicate by a "flag" that the matrix $c(i, j)$ is symmetric; hence only the upper right triangle is given explicitly in the compressed data library. These various aspects of the Boxer format are illustrated by a simple example in Fig. 1. Here a, b, c, and $d$ are arbitrary, non-zero, unequal data values.

Original Data Set

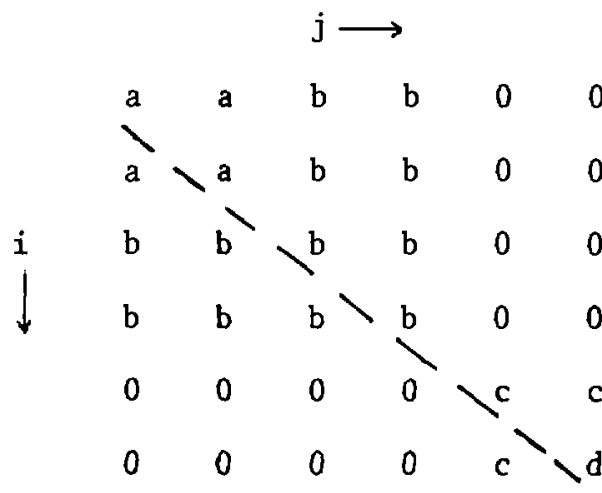

Boxer Format, Symmetry Flag off

$\begin{array}{rrrrrrrrr}a & b & b & 0 & c & d & & & \\ -2 & -2 & 8 & -4 & 8 & -4 & -2 & 5 & -1\end{array}$

Boxer Format, Symmetry Flag On
a $\quad$ b $\quad c \quad d$
$\begin{array}{lllll}-2 & -2 & 14 & -2 & -1\end{array}$

Fig. 1. Illustration of Boxer format. 
Before the data values are tested in PRESS to see if they are indeed equal, the NJOY utility SIGFIG is called to round off the trailing digits that would not appear on the formatted output anyway. Relative covariances, for example, are written to NOUT in 1P8E10.3 format, which has only four significant figures. Thus, the three data values $0.036126,0.036130$, and 0.036134 would all be judged to be "equal" by this logic.

\section{Generation of Plots}

The COVR plot mode, which is entered by specifying NOUT $=0$, is used to generate publication-quality plots of multigroup covariance data from a cardimage or binary ERRORR output file. Examples of plots produced by COVR can be seen in Figs. 2 and 3 of Chapter XIX of this report. Many more examples can be found in Ref. 3 .

In addition to their usefulness in preparing publications, the plots have proven to be a useful tool for checking the reasonableness and mechanical correctness of new covariance evaluations. One can, for example, execute ERRORR and COVR in tandem, using the evaluator's energy grid as the "user's" group structure (ERRORR input option $I G N=19$ ). The output of such a run is a series of plots showing all important features of the covariance evaluation.

As can be seen in the examples mentioned above, each plot contains a shaded "contour map" of the correlation matrix. Positive-correlation regions are shaded with parallel straight lines, while negative correlations are indicated by cross-hatching. The plots also contain two semilogarithmic curves giving the energy dependence of the associated standard deviation vectors. One of the vector plots is rotated by 90 degrees, so that the logarithmic energy grids for the vector plots can be aligned with the corresponding grids for the matrix plot:

This type of plot presents the covariance data in a more lucid manner than most alternative plotting packages. However, use of this option does require the availability of the proprietary plotting-software package DISSPI.A. ${ }^{5}$ Only a few of the DISSPLA subroutines are actually called (curve plotting, shading, Greek lettering, and subplot centering and rotation), so that conversion to other plotting packages is probably quite feasible. Users who do not have access to DISSPLA and who do not wish to convert COVR to another system can obtain a set of code corrections from the code authors to simply remove the plot option. 
Just as in the library mode, the material and reaction coverage of the sequence of plots generated in the plot mode is determined by the reaction pairs specified by the user on input Card 4. Other input, specific to the generation of plots, is described in Section $D$. We next discuss some aspects of the plot-generation coding.

After a covariance matrix is read in SUBROUTINE COVARD, it is converted to a correlation matrix in SUBROUTINE CORR. (See the discussion of such conversions in Section B of Chapter XIX of this report.) The correlation matrix is then scanned in SUBROUTINE MATSHD to find a set of boundary curves that divide $E_{x}-E_{y}$ energy space into a small number of connected regions of nearly constant correlation strength. Here the phrase "nearly constant" refers to the subdivision of the range of possible correlation values $(-1.0$ to +1.0$)$ into a number of equal-width bands, the fineness of the subdivision being controlled by the user-input parameter NDIV. Two regions, then, have "nearly equal" correlations if the correlations fall into the same band. As each boundary curve is located, the region within is shaded (with a line density proportional to the band correlation magnitude) with a single call to the DISSPLA areashading routine SHADE.

The algorithm used to find the maximum extent of a region of nearly constant correlation is best described by referring to a simple example such as that in Fig. 2. After locating the upper left corner of a new correlation region, for example at point a in the figure, the search proceeds as far as possible down the first "column" ( $E_{y}$ held constant) to point $b$. New columns are scanned in the same way: $c-d, e-f, g-h$. Having found that the pattern does continue into column $e-f$, for the sake of simplicity, the algorithm ignores the possibility of additional, disjoint, continuations of the pattern elsewhere in the same column (such as at $e^{\prime}-f^{\prime}$ ), and region $I$ is ended at $h$. (Region II will be found and correctly shaded at a later stage of the calculation.)

Multiple calls to the SHADE routine usually produce a discernible discontinuity in the shading pattern (along the dashed line in Fig. 2). This is not really a problem, but the effect is occasionally noticeable in applications. See, for example, Fig. 80 of Ref. 3. Note there that, while c-shaped regions require multiple calls to SHADE, u-shaped regions do not. 


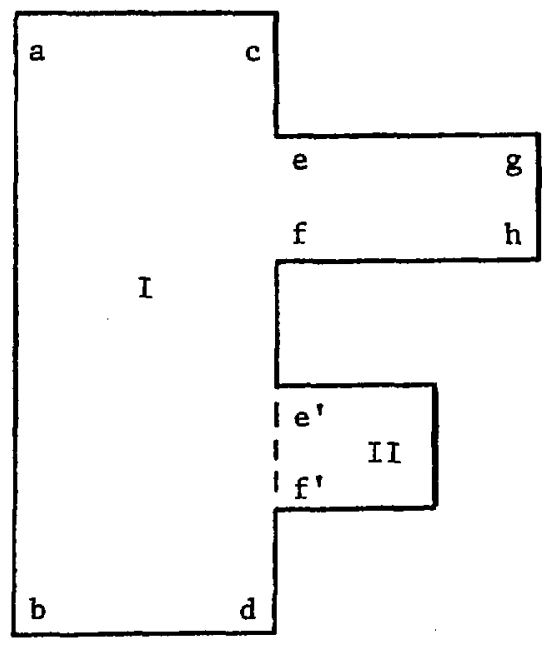

Fig. 2. Pattern - Search Logic in MATSHD.

As the plot option is presently coded, the entire correlation matrix must reside in core during the pattern-search operation just described. This fact, coupled with the fact that the availability of central memory is already 1imited in COVR because of the space occupied by the DISSPLA subroutines, means that only relatively coarse group structures are permitted in the plot option. On the CDC 7600, for example, the container array in COMMON/STOREC/ is currently dimensioned at only 6000 words, and this permits plotting of data sets with up to 42 groups. On a CRAY-1, with about an order of magnitude more central memory, this problem would essentially vanish.

\section{Input Instructions for CoVR}

As an aid to discussions of the user input to COVR, we list below the input instructions that appear as comment cards at the beginning of the current version of this module. This module has been changed fairly often in recent years, so it is always advisable to consult the coment-card instructions on the version of the code actually being used and not to rely on the instructions published in any document, including this one. Following the listing are further remarks on several of the input items, which supplement the coment cards below. See especially the lengthy discussion of the parameters on Card 4. 


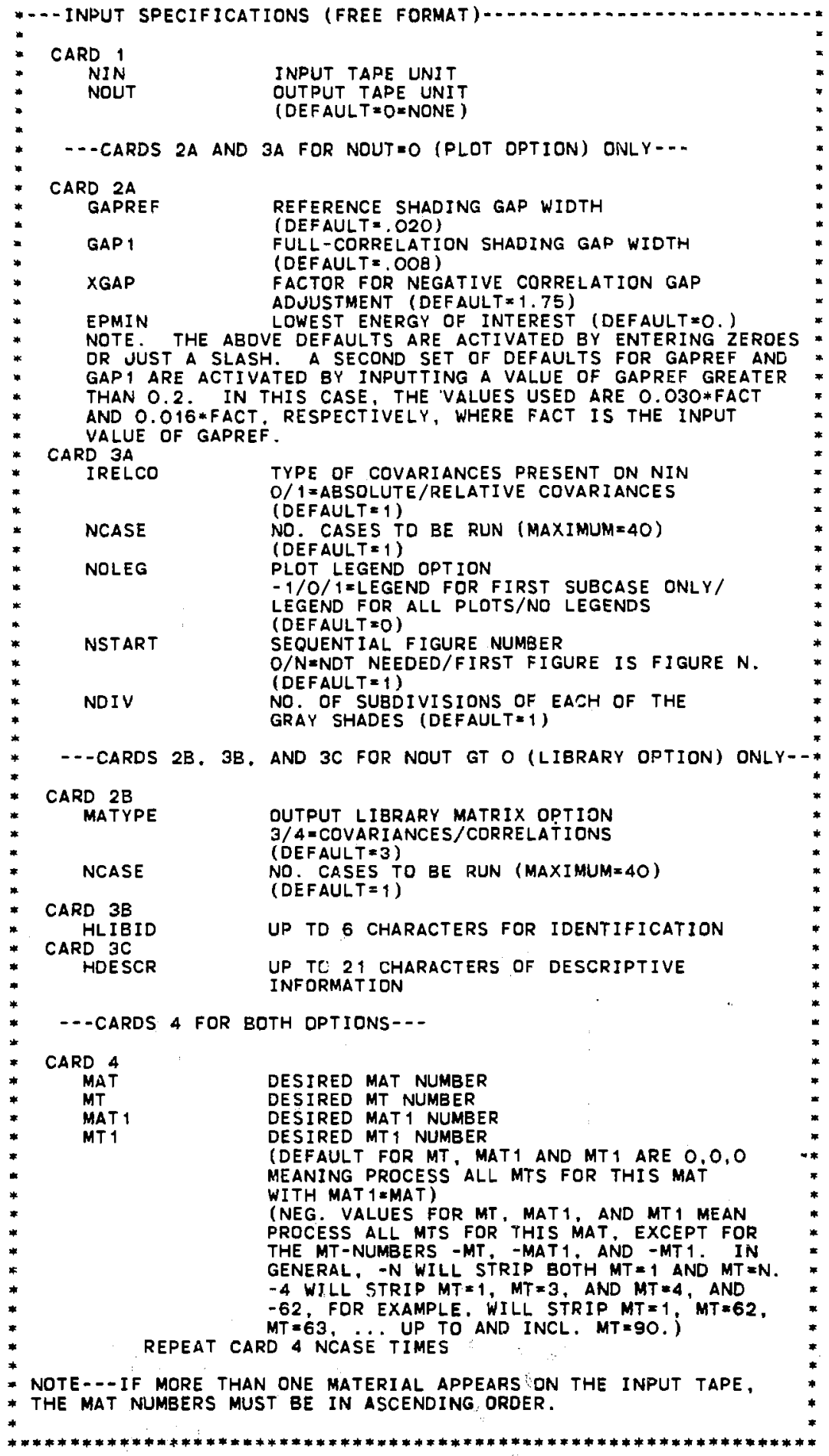


GAPREF, GAP1, XGAP... These parameters may need to be adjusted away from the default values to achieve good results with some plotting devices.

EPMIN... This parameter is used to eliminate uninteresting energy regions from the correlation and standard deviation plots, or to display high-energy regions with greater resolution.

IRELCO... Must match the value used in the ERRORR run that produced the covariances to bie plotted.

NCASE... This is the number of "cases," or occurrences of Card 4. "See the discussion of input parameters MAT, MT, MAT1, and MT1 below. Present1y, NCASE is limited to 40 .

NOLEG... "Legend" here refers to the gray-shading scale (key) as well as the figure caption. NOLEG $=1$ is used as a "rough draft" mode to display plots quickly.

NSTART... Unless NSTART $=0$, the plots are assigned a sequentiai figure number, beginning at NSTART, and a "List of Figures" is drawn on the final plot frame:

NDIV... One gray-shade step equals 0.20 in correlation magnitude if NDIV $=1$. Finer gradations are possible with NDIV greater than 1. The plots appearing in Figs. 2 and 3 on pages 22 and 24 of this report were generated with NDIV $=2$.

HLIBID... This is normally the Hollerith "name" of the output covariance library. It is written on the header cards present at the beginning of each out. put data block.

HDESCR... This normally contains information on where and when the library was produced. Also written on the data header cards.

MAT, MT, MAT1, MI1... The information contained in the output Iibrary or plot file is controlled by means of these parameters on input Card 4 . If MT is positive, then a single covariance watrix for reaction (MAT,MT) with reaction 
(MAT1,MT1) will be read from NIN and processed. On unit NIN (and, in the library option, on unit NOUT), the rapidly varying, or "column," index is the group index of (MAT1,MT1), and the slowly varying, or "row," index is the group index of (MAT,MT). If MAT1 is different from MAT, COVR will expect to find separate materials (produced by separate ERRORR. runs) for both MAT and MAT1 on NIN. The MAT numbers must occur on NIN in ascending order. In the case of positive MT, the entries MAT1 $=0$ or MT1 $=0$ are shorthand for MAT1 = MAT or MT1 = MT, respectively. If, on the other hand, MT is zero, negative, or defaulted, Card 4 becomes a kind of macro-instruction that is expanded by SUBROUTINE EXPNDO into a request for many MT-MT1 pairs for the material MAT, with MAT1 $=$ MAT. If, for example, Card 4 contains the entry

\section{MAT/}

or, equivalently,

MAT 000 ,

first the cross-section file, $M F=3$, for material MAT is read from the input covariance "tape" on unit NIN to obtain the list of reactions present. Then, al1 possible reaction combinations MT-MT1 are formed in ENDF/B order. Thus, for example, if the reactions present are $M T=1,2,3$, and 16 , then the behavior of the code is the same as if the following input were specified:
MAT 1010
MAT 1002
MAT 1003
MAT 1016
MAT 2002
MAT 2003
MAT 20016.
MAT 303
MAT 3016
MAT $16 \quad 0 \quad 16 \quad$

Because of the clear labor-saving advantage of this feature, especially if there are many reactions, enhancements have been added to permit its use in the additional situation in which many, but not all, combinations are desired. As discussed in some detail in the input instructions, it is possible to "strip" selected reactions out of the list before the MT-MT1 combinations are formed. For example, if the reactions present are once again $1,2,3$, and 16 , then a Card 4 containing MAT $-3 /$ would produce the same output as the following three cards: 
MAT 2002

MAT 20016

MAT 16016 .

The stripping of the higher inelastic levels, mentioned in the input instructions, is useful because ERRORR automatically resets the highest user-group energy to $20 \mathrm{MeV}$ whenever IREAD $=0$, and this often results in the inclusion of unwanted high-threshold reactions on the ERRORR output file.

For one of several different reasons, a requested reaction pair (MAT,MT; MAT1,MT1) may be absent from the COVR output. The usual reason that this occurs is that the normal IREAD option for the ERRORR module is IREAD $=0$, and this results in the generation of an output matrix for every possible reactionpair combination. For those combinations that do not occur in the ENDF/B evaluation, the ERRORR output matrix contains only zeroes. These "null" matrices are omitted at the COVR output stage, in both the library and plot modes.

Additionally, in the plot mode, the correlations may be non-zero, but everywhere less than $0.2 / \mathrm{NDIV}$ in absolute magnitude. In this case the entire correlation plot would consist of a single blank region. These rather uninteresting plots having "small" correlations are also omitted from the plot file.

A final, similar category is the class of "empty" plots. Because DISSPLA uses parallel lines to achieve a "gray" effect in the SHADE routine, there is clearly some lower limit, for a given value of the correlation magnitude, on the physical size of a region that can be sensibly shaded. CoVR does not attempt to shade regions that are smaller than this limit. If a correlation matrix does contain some "plottable" data (magnitudes exceeding 0.2/NDIV), but all "plottable" regions are smaller than the size limit discussed above, then an empty plot will be generated on the plot file. As a convenience in discarding these empty plots at the time a report is produced, no caption is written on such plots and the figure number is not advanced. Also, these plots are omitted from the "Iist of Figures" prepared at the end of a plot run. (See the discussion of user-input parameter NSTART.)

In the library mode, each omission of a requested, but "null," matrix is noted on the OUTPUT file with an informative diagnostic. In the plot mode, a sumary table is printed at the end of the run to identify all requested antrices that were onitted because they were "null" or "snall," as well as those that were plotted, but "eapty." 


\section{E. COVR Example Problem}

In this section we discuss the production of a particular CoVR output library, both to illustrate the input and as a supplement to the general discussion of Boxer-format libraries in the Section B above. On page 31 of this report we gave the complete NJOY input for producing a 7-reaction, 30-group covariance library in ERRORR output format for ${ }^{12} \mathrm{C}$. By appending the following lines to that input (just before the $*$ STOP card), one can produce, in addition, a 2-reaction COVR output library in Boxer format.

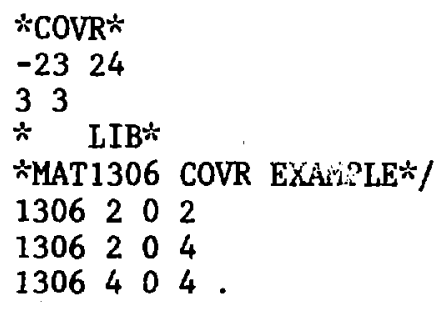

The resulting library occupies only 49 lines and is listed below.

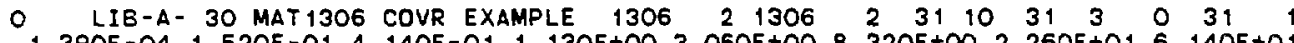

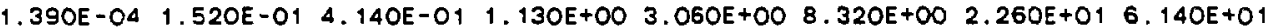
$1.67 O E+02 \quad 4.54 O E+O 2 \quad 1.235 E+O 3 \quad 3.35 O E+O 3 \quad 9.120 E+03 \quad 2.4 B O E+04 \quad 6.760 E+04 \quad 1.84 O E+O 5$

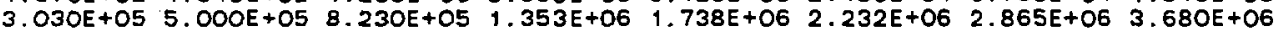
$6.070 E+06 \quad 7.790 E+06 \quad 1.000 E+07 \quad 1.200 E+07 \quad 1.350 E+07 \quad 1.500 E+07 \quad 1.700 E+07$ $-1-1-1-1-1-1-1-1-1-1-1-1-1-1-1-1-1-1-1-1-1-1-1-1-1-1-1-1-1$ $-1-1-1-1-1$

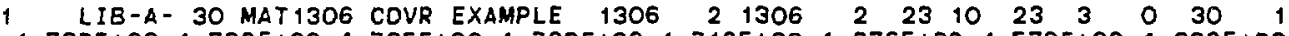
$4.739 E+O O \quad 4.738 E+O O \quad 4.735 E+0 O \quad 4.729 E+\infty \quad 4.712 E+O 0 \quad 4.676 E+\infty \quad 4.579 E+00 \quad 4.332 E+\infty O$ $4.002 E+O O \quad 3.619 E+O O \quad 3.103 E+O O \quad 2.477 E+\infty O \quad 1.998 E+\infty \quad 1.820 E+\infty O \quad 1.710 E+00 \quad 2.256 E+\infty O$ $1.447 E+00$ 9.921E-01 8.223E-01 7.627E-01 8.631E-01 8.228E-01 8.845E-01

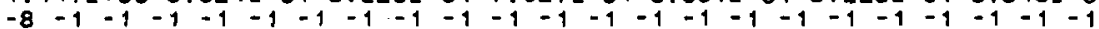

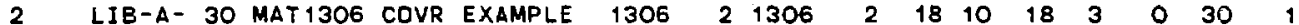
$2.000 E-03 \quad 1.964 E-03 \quad 4.583 E-03 \quad 3.822 E-03 \quad 4.583 E-03 \quad 4.288 E-03 \quad 3.730 E-03 \quad 4.141 E-03$ 6.5O1E-03 1.011E-02 9.558E-03 9.981E-03 1.806E-O2 1.854E-02 3.296E-02 3.760E-O2 $4.255 \mathrm{E}-\mathrm{O} 27.632 \mathrm{E}-\mathrm{O} 2$

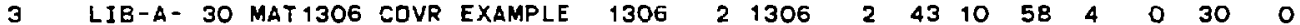

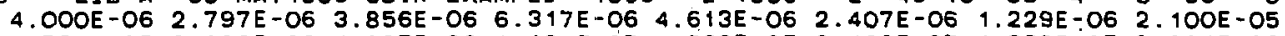

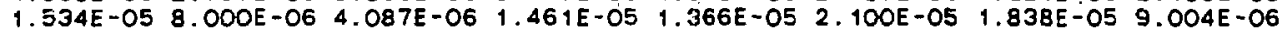
$1.391 E-05 \quad 8.934 E-06 \quad 1.715 E-05 \quad 4.447 E-06 \quad 4.227 E-05 \quad 4.373 E-05 \quad 2.446 E-05 \quad 1.980 E-05$ $1.223 E-05 \quad 1.022 E-04 \quad 6.116 E-05 \quad 4.048 E-05 \quad 2.500 E-05$ 9.136E-05 $4.572 E-05$ 9.962E-05 $\begin{array}{llllllll}6.641 E-05 & 6.308 E-05 & 3.260 E-04 & 1.614 E-04 & 3.437 E-04 & 1.086 E-03 & 4.250 E-04 & 1.413 E-03\end{array}$ $7.052 E-041.811 E-035.825 E-03$

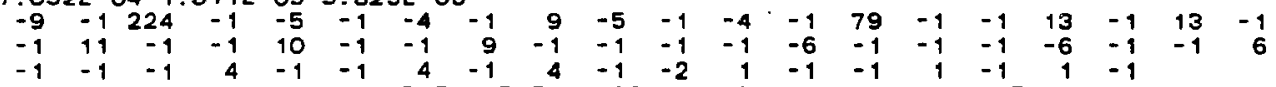

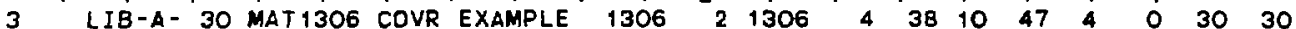
-1. 106E-04-4.897E-05-3.047E-05-2. 164E-05-2.630E-05-2 .337E-05-2. 192E-05-2. 26 1E-04 - 1.001E-04-6.228E-05-4.423E-05-5. 376E-05-4.777E-05-4 . 48 1E-05-1.441E-03-2 .659E-04 $-1.571 E-04-1.210 E-03-1.305 E-03-4.021 E-04-1.131 E-03-6.462 E-04-8.562 E-04-2.261 E-04$ $-1.001 E-04-6.228 E-05-1.922 E-03-9.14 O E-04-8.121 E-04-7.520 E-04-3.040 E-03-1.348 E-03$ $-1.517 E-03-3.460 E-03-4.423 E-05-5.376 E-05-4.777 E-05-1.044 E-02$

$\begin{array}{rrrlllllllllllllllll}623 & -1 & -1 & -1 & -1 & -1 & -1 & -1 & 23 & -1 & -1 & -1 & -1 & -1 & -1 & -1 & 53 & -1 & -1 & -1 \\ 27 & -1 & -1 & -1 & 27 & -1 & -1 & -1 & 27 & -1 & -1 & -1 & -1 & -1 & -1 & 27 & -1 & -1 & -1 & 28\end{array}$ $\begin{array}{lllllll}-1 & -1 & 27 & -1 & -1 & -1 & -1\end{array}$

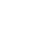




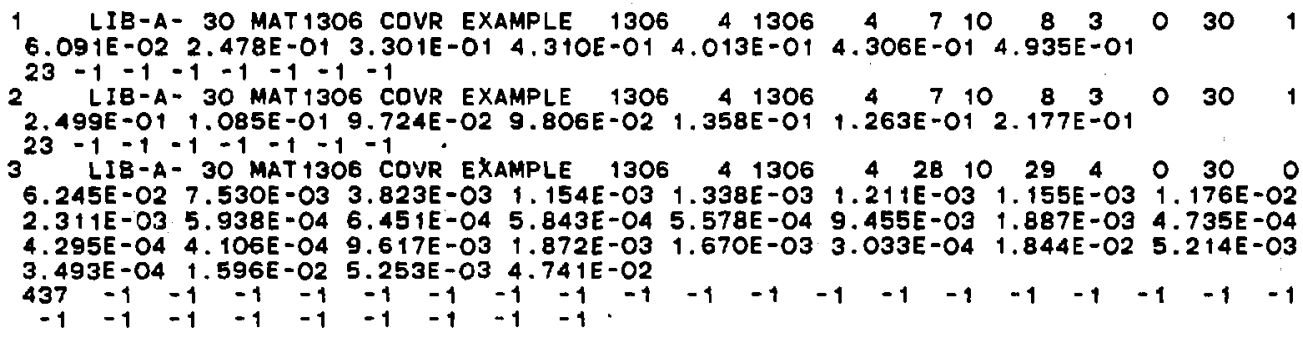

Note that the header card at the start of each data block contains an integer ITYPE, specifying the type of data contained in the current block, a 12-character library name (" LIB-A- 30" in this case), 21 characters of user-supplied descriptive information, MAT, MT, MAT1, MT1, and a set of 7 integers. The meaning of the various values of ITYPE is as follows: $0=$ group boundaries, $1=$ cross sections, $2=$ standard deviations, $3=$ covariances, $4=$ correlations. The library name is generated within COVR by adding either "-A-" (for a covariance library) or "-B-" (for a correlation library), together with the number of energy groups, to the user-supplied library name HLIBID. (The ERRORR input option IGN $=3$ used in this example specifies a built-in 30-group structure). The final seven integers on the header card indicate the number and format of the data values, the number and format of the "control" integers (called " $m$ " and "- $n$ " in the discussion in Section B), a data "paging" flag, and the dimensions of the reconstructed data array, $c(i, j)$.

In order to clarify the process of reconstructing a full matrix from data in Boxer format, we list in the Appendix below a short retrieval program, BOXR. With very few modifications, this program could be incorporated into a sensitivity analysis program, for example, to allow direct access to covariance libraries $^{6}$ in this compact format. Alternatively, it could be used to translate COVR libraries into other desired forms. In any case, an examination of the retrieval program should clarify the meaning of the various integer parameters on the header cards in a COVR output library. 
F. Error Messages in COVR

COVR:HEQUESTED TOO MANY CASES.

NCASE is limited to 40 . Note that a "case" may be either a single reaction pair or a whole series of reaction pairs for one material. See input instructions for Card 4 and the comments that follow.

EXPND0* $\div$ STORAGE EXCEEDED.

Either there is insufficient space in array ISCR to store the cross sections or the total number of reaction pairs in the current case exceeds 300. In the latter instance, it may be necessary to specify the reaction pairs individually (up to 40 per run) in a long series of runs, and then concatenate the numerous output files to form a single COVR output file.

CORR

Should not occur.

COVARD:-2:STORAGE EXCEEDED.

Insufficient space in array ISCR to store the multigroup data from NIN, the ERRORR output file.

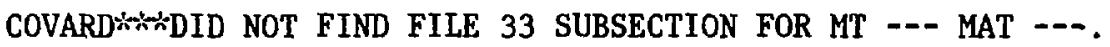

Requested reaction $y$ of the current $x-y$ pair is not found on NIN.

TRUNC + BAD DATA.

Either all cross sections are very small (less than XSLIM $=10^{-4}$ barn) or EPMIN is larger than the highest group boundary.

MATSHD

Maximum number of correlation patterns is 1000 . Reduce NDIV to 1 or raise EPMIN, in order to reduce the complexity of the correlation plot.

MATSHD:*'*STORAGE EXCEEDED.

NWIG $=2 *($ IXMAX +1$)+600$ words are available for storing the boundary curve of a constant-correlation region. This will not be exceeded for any practical number of groups IXMAX.

LEVEL $:-2$ COEFFICIENT $=---$ OUT OF RANGE.

The absolute magnitude of a computed correlation coefficient is greater than 2. The input covariance file may be faulty.

HISTO

Should not occur. 


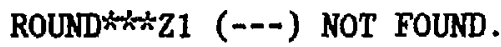

Should not occur.

FINDS $\dot{2} \dot{2}$ MAT -.- MF -.- MT --- NOT ON TAPE.

Requested reaction $x$ of the current $x-y$ pair is not found on NIN.

PRESS

Should not occur.

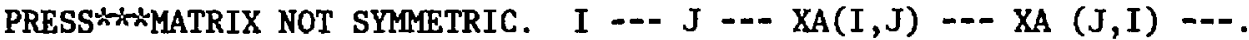

Symmetric-matrix format requested for a matrix that is asymmetric. Should not occur.

SETFOR

Should not occur.

G. Input/Output Units

The following logical units are used.

10 NIN in CORR and COVARD, NSCR in other routines. Used to extract either one or two (if MAT1 $\neq$ MAT) materials from the input covariance tape.

11 NSCRl in the plot mode. Used to document null and small covariance matrices and empty plots.

$11 / 12$ NSCR1/NSCR2 in the library mode. In COVARD, the input covariances for the current reaction pair are read from unit 10 and written to NSCR2 $(=12)$. If the output library is to contain correlation coefficients (MATYPE = 4), then in CORR the covariances are read from NSCR2, and the calculated correlations are written to NSCR1 $(=11)$. If output covariances are requested (MATYPE $=3$ ), the value of NSCR1 is simply reset to NSCR2 $(=12)$. In either case, PRESS reads the data from unit NSCR1 and writes the compressed data to NOUT.

20-99 User's choice for NIN and NOUT.

Unit 10 has the same mode as NIN. NOUT, if used, is always formatted. Unit 11 is formatted in the plot option and binary in the library option. Unit 12, if used, is always binary. 


\section{G. References for COVR}

1. S. A. W. Gerst1, "SENSIT: A Cross-Section and Design Sensitivity and Uncertainty Analysis Code," Los Alamos Scientific Laboratory report LA-8498MS (August 1980).

2. Mark J. Embrechts, "SENSIT-2D: A Two-Dimensional Cross-Section Sensitivity and Uncertainty Analysis Code," Los Alamos National Laboratory report LA-9515-MS (October 1982).

3. D. W. Muir and R. J. LaBauve, "COVFILS: A 30-Group Covariance Library Based on ENDF/B-V," Los Alamos National Laboratory report LA-8733-MS (ENDF-306) (March 1981).

4. D. W. Muir, R. E. MacFarlane, and R. M. Boicourt, "Multigroup Processing of ENDF/B Dosimetry Covariances," Proc. 4th ASTM-EURATOM Symp. on Reactor Dosimetry, Gaithersburg, Maryland, March 22-26, 1982, NUREG/CP-0029 (CONF820321), p. 655 (1982).

5. "DISSPLA (Display Integrated Software System and Plotting Language): User's Manual, Version 9.2," Integrated Software Systems Corporation, 4186 Sorrento Valley Blvd., San Diego, California 92121.

6. D. W. Muir, "The COVFILS-2 Library of Neutron Cross Sections and Covariances for Sensitivity and Uncertainty Analysis," in E. D. Arthur and A. D. Mutschlecner, Comps., "Applied Nuclear Science Research and Development Semiannual Progress Report," Los Alamos National Laboratory report LA10288-PR (January 1985), pp. 38-42. 


\section{APPENDIX}

\section{RETRIEVAL PROGRAM FOR COVR OUTPUT LIBRARIES}

PROGRAM BOXR (INPUT, TAPE5 = INPUT, TAPE20, TAPE2 1, TAPE22)

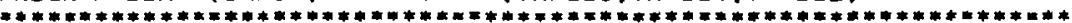
FUNCTION OF PROGRAM. READ DATA FROM UNIT NIN IN THE COMPRESSED *BOXER* FORMAT PRODUCED BY THE COVR MODULE OF NUDY, AND LOAD THE FULL. RECONSTRUCTED MATRIX INTO C(I,U). THEN WRITE THE RESULT ON UNIT NOUT IN HIGH-TO-LOW ENERGY ORDER. FAILURE TO FIND A REQUESTED DATA SET RESULTS IN AN ERROR STOP.

ITYPE DATA TYPE REQUESTED

* - 1 , TO WRITE A TABLE OF CONTENTS OF NIN, IN THE FORMAT DF BOXR INPUT INSTRUCTIONS, ON UNIT NTAB.

= 0 . FOR GROUP BOUNDARIES.

= 1. FOR CROSS SECTIONS.

- 2, FOR STANDARD DEVIATIONS

= 3. FOR COVARIANCE MATRIX.

= 4, FOR CORRELATION MATRIX, OR (IF MT I IS ZERO) TRANSFER MATRIX FROM COVFILS2.

ITYPEH VALUE DF ITYPE ON CURRENT DATA HEADER CARD.

(MAT, MT, MAT 1,MT 1) REQUESTED REACTION PAIR.

(MATH, MTH, MATTH, MT 1H) CURRENT REACTION PAIR.

(XVAL(IV), IV=1, NVAL) DATA VALUE ARRAY IN THE *BOXER* FORMAT. NVMAX

( I CON (IC) $, I C=1, N C O N)$

MATA VALUE ARRAY IN THE *BOXER*

NCMAX

I ROW INDEX OF MATRIX $C(I, J)$, NORMALLY THE ENERGY GROUP OF THE REACTION (MAT, MT):

NROW NUMBER OF ROWS IN C (I.J).

NROWH VALUE OF NROW ON DATA HEADER CARD.

NROWM CONTINUATION FLAG, = O FOR FINAL DATA BLOCK OF CURRENT REACTION PAIR.

$J$ COLUMN INDEX OF $C(I . J)$, FOR MATRIX DATA THE ENERGY GROUP OF THE REACTION (MAT 1 MT 1 ), $\approx 1$ FOR VECTORS.

NCOL NUMBER OF COLUMNS IN C $(I, U)$.

NCOLH VALUE DF NCOL ON DATA HEADER CARO, = 0 IF $C(I, J)$ IS A SYMMETRIC MATRIX REPRESENTEO IN THE *BOXER* FORMAT BY JUST THE UPPER RISHT TRIANGLE (J.GE.I).

NGMAX MAXIMUM ALLOWABLE $\geq$ LUE OF NROW AND NCDL

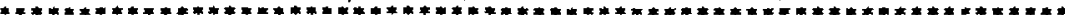

DIMENSIDN IVFT ( 3 ), ICFT (3), IA (9), IB(9)

DIMENSION C (200,200), CR (200), XVAL (880), ICON(900)

DATA NGMAX /200/, NVMAX /880/. NCMAX /900/, IDASH /4H----/

C

DATA NINPUT $/ 5 /$, NIN $/ 20 /$. NOUT $/ 21 /$, NTAB $/ 22 /$, IZERO $/ 0 /$

C **\#EAD USER-SUPPLIED REACTION-TYPE AND MAT-MT INFORMATION.

10 READ (NINPUT. 190) I TYPE, MAT, MT, MAT 1 , MT

INPUT IS TERMINATED BY ENTERING $(0,0)$.

IF (ITYPE.GE.O.AND.MAT.EQ.O) STDP

C

***RETRIEVE REQUESTED DATA FROM UNIT NIN.

20 READ (NIN, 210) ITYPEH, (IA I ), I = 1,9), MATH, MTH, MAT 1H, MT IH, NVAL, NVF

1 .NCDN, NCF , NROWM, NRDWH, NCOLH

$\operatorname{CCDC}$

IF (EDF(NIN)) 900,30

$\operatorname{CCDC}$

30

IN COVFILS2, ITYPEH=9 IS USED AS A TERMINATOR.

IF (ITYPEH.EO.9) 60 TO 900

IF (ITYPE.EQ. - 1. AND.MATH.EQ.O) MATH=1

IF (ITYPE. EQ. - 1. ANO. IA ( 2 ). NE. IOASH)

1 WRITE (NTAB, 190) ITYPEH, MATH, MTH, MAT IH, MT IH

IF (NVAL.GT.NVMAX) STOP 3

IF (NCON. GT. NCMAX) STOP

C SET FORMATS. THEN READ BOXER DATA.

CALL SETFOR (NVF, NCF, NOUT, IVFT, ICFT)

IF (NVALIGT .O) READ (NIN, IVFT) (XVAL (I) I I = . NVAL)

IF (NCDN.GT.O) READ (NIN, ICFT) (ICON(I),I=1,NCON) 


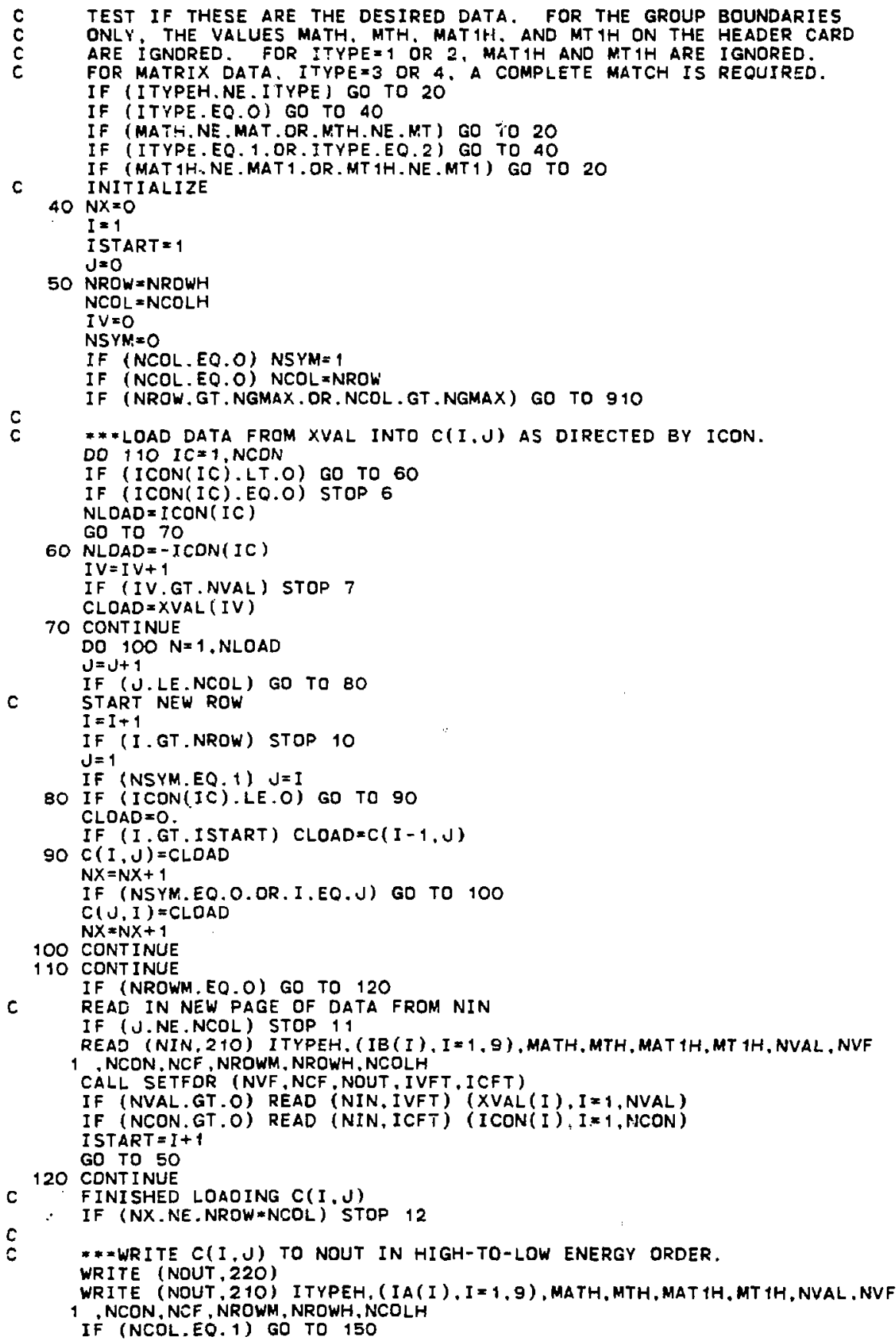




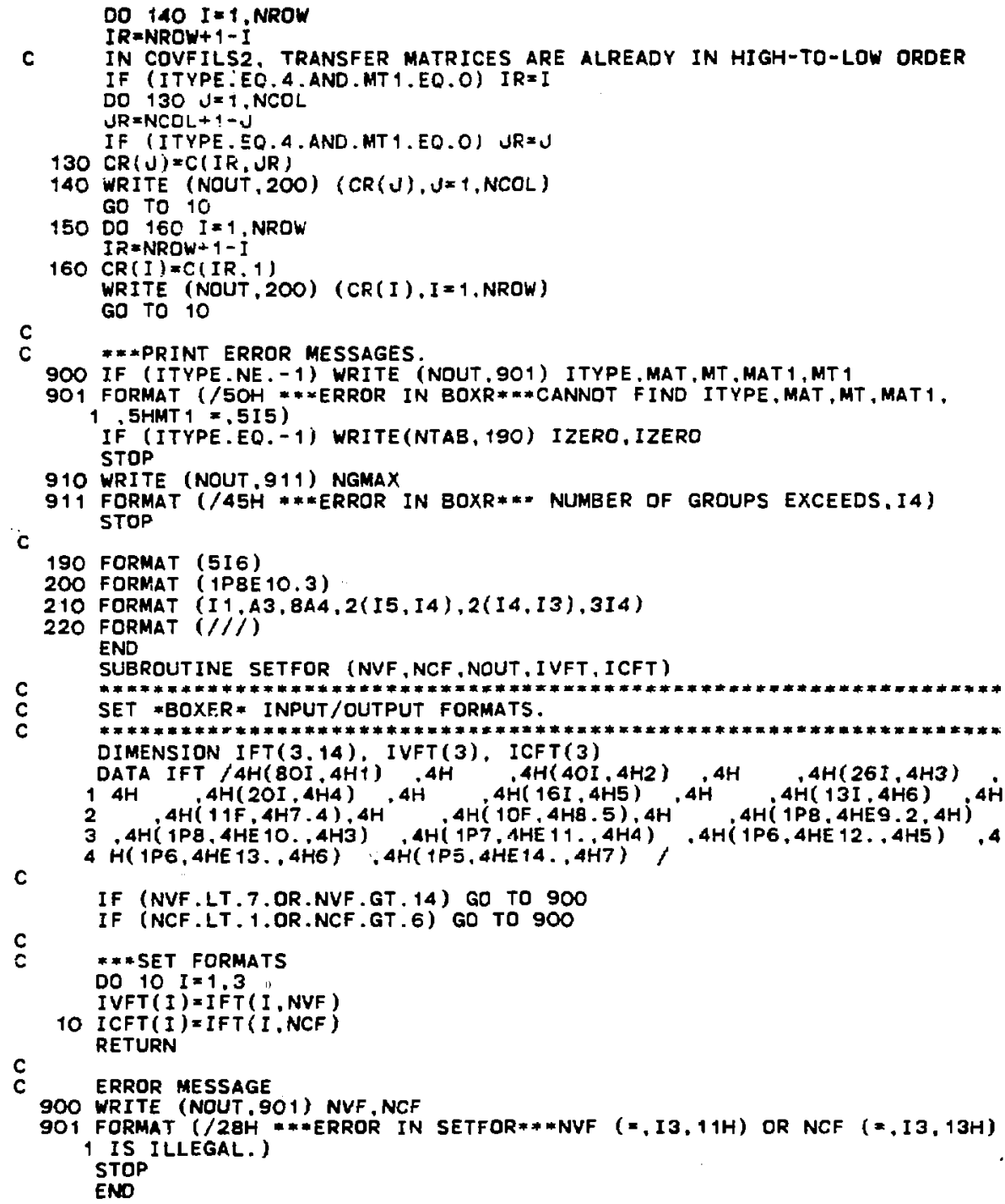


